\title{
Review Paper: \\ Urban Mental Health Initiative: Developing Interdisciplinary Collaboration, the Role of the Family Physician in Management of Severe Mentally III Patients
}

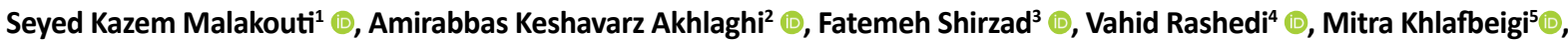 \\ Maziar Moradi Lakeh ${ }^{6}$ (1), "Ahmad Hajebi ${ }^{7}$ 이
}

\author{
1. Mental Health Research Center, School of Behavioral Sciences and Mental Health (Tehran Institute of Psychiatry), Iran University of Medical Sciences, Tehran, Iran \\ 2. Mental Health Research Center, Iran University of Medical Sciences, Tehran, Iran. \\ 3. Spiritual Health Research Center, Iran University of Medical Sciences, Tehran, Iran. \\ 4. School of Behavioral Sciences and Mental Health (Tehran Institute of Psychiatry), Iran University of Medical Sciences, Tehran, Iran. \\ 5. Department of Occupational Therapy, School of Rehabilitation Sceinces, Iran University of Medical Sceinces, Tehran, Iran. \\ 6. Family Health Research Center, School of Medicine, Iran University of Medical Sciences, Tehran, Iran. \\ 7. Department of Psychiatry, Research Center for Addiction and Risky Behaviors (ReCARB), Iran University of Medical Sciences, Tehran, Iran.
}

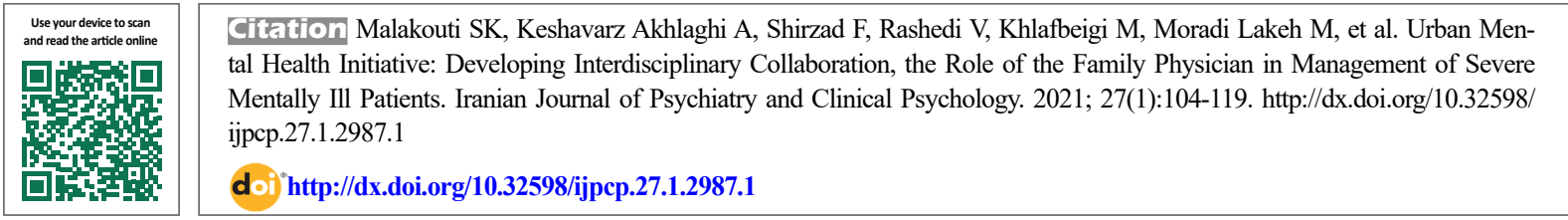

Received: 23 Nov 2019 Accepted: 20 Jun 2020 Available Online: 01 Apr 2021

Key words:

Mental health, Community-based services, Urban mental health, Family physician

\section{A B STRACT}

Objectives Mental health program in Iran has been approved since 1988 and has been integrated into the primary care system especially in rural areas, and has been developed greatly over the past few decades. Due to demographic changes in the country's population and the rapid expansion of cities, there is a need for a coherent plan to provide mental health services in urban areas. This study aims to provide a practical model for mental health services in patients with severe psychiatric disorders in urban areas. Methods This study was conducted based on reviewing literature, using the experiences of other countries, the model of the World Health Organization, the services available in Iran, description of the responsibilities of beneficiary organizations, collaboration between the Ministry of Health and various organizations responsible for providing mental health, and using the opinios of national and international experts. In this model, urban mental health services are estimated for a population of 100,000 people. Results The developed model proposed to provide immediate psychiatric services with more coherence, increase the training and skills of the staff of 110, 115, 1480, and 123 hotlines, and determine a space for hospitalization of 3-5 emergency patients next to the public hospital. Depending on the number of patients per 100,000 population, there would be a need for community-based health service providers, including 2-3 rehabilitation teams for home visits (each cover 80 patients), and 3 daycare centers to provide services to 40 patients. In case of providing community-based services, there would be a need for 12 beds for acute psychiatric patients and 5 beds for mid and long term rehabilitation. Moreover, vocational rehabilitation services should provide to 50 patients, and two apartments with an area of about $80 \mathrm{~m}^{2}$ would be needed to accommodate about 8 patients. The provision of above mentioned services would require equal participation of beneficiary organizations. The family physician would play an essential role in follow-up of patients under the constant supervision of specialists.

Conclusion To achieve the desired model of urban mental health services so that it can provide comprehensive services to patients at different levels from prevention and care to treatment and rehabilitation, there is a need for collaboration between organizations providing these services. In this model, the use of existing services imposes lower financial burden on the country's mental health management system

\section{* Corresponding Author:}

Ahmad Hajebi, MD.

Address: Department of Psychiatry, Research Center for Addiction and Risky Behaviors (ReCARB), Iran University of Medical Sciences, Tehran, Iran.

Tel: +98 (915) 5120609

E-mail: hajebi.a@iums.ac.ir 


\section{Extended Abstract}

\section{Introduction}

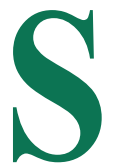

tudies on the integratation of mental health into primary health care in Iran have been conducted since 1986, which was later implemented successfully within the Rural Family Physician Program in 1988. In urban areas, there is no structured mental health care system and the private sector provides health care services mostly focused on the biomedical model of care. Due to demographic changes in Iran's population, urban sprawl, and the changes in the urban/rural proportion, the need for a coherent plan to provide mental health services in urban areas is felt more than ever. This study aims to investigate the feasibility of establishing the urban mental health care system for patients with severe psychiatric diseases based on collaboration between organizations in Iran.

\section{Materials and Methods}

The study reviews the integrated plan for urban mental health services in Iran. First, a systematic review was conducted to get acquainted with the models of providing urban mental health services worldwide, including the World Health Organization (WHO)'s model in the field of mental health care. Then, the situational analysis of the services provided in Iran was carried out using the WHO Assessment Instrument for Mental Health Systems (WHOAIMS). Legal duties of beneficiary organizations, and the Inter-sectoral collaboration of the Ministry of Health and beneficiary organizations were reviewed along with seeking for the opinions of international and national experts using the Delphi method. The model estimates the need for mental health services in cities with 100,000 population.

\section{Results}

The prevalence of mental diseases in Iran was similar to the global rate. The most prevalent mental disease was major depressive disorder followed by substance abuse. The number of male and female patients with mental diseases was not equal. The proposed model was mainly for severe mental diseases such as severe depression, bipolar mood disorder, and the spectrum of psychotic disorders that account for the highest number of admissions to psychiatric hospitals. According to the results of the literature review and based on the opinions of experts, the urban mental health care system should provide the following services to psychiatric patients: (a) Integrated emergency psychiatric services in the form of crisis hotlines, mobile crisis intervention teams, and inpatient wards. Some of the crisis hotlines that are currently operational in Iran are the Police (Dial 110), Medical Emergency (Dial 115), and Social Emergency (Dial 123). There is no need to provide more similar services, especially new crisis hotlines and mobile crisis intervention teams; (b) Short- and long-term hospitalization services. Considering a point prevalence of $22 \%$ for psychiatric disorders in Iran according to the results of the systematic review and meta-analysis, a minimum estimate of $3 \%$ for the annual prevalence of severe psychiatric disorders was considered as the criterion for calculating the number of beds and providing other future services. Based on the made calculations, there was a need for 12 beds for acute psychiatric diseases and 5 beds for shortand long-term hospitalization for a population of 100,000 in case of providing community mental health services; (c) Home visit services. The psychiatric patients can be classified into two groups according to their disease severity: a group requiring more follow-up care and a group with the need for less follow-up care. For the first group, home visit services are currently provided by the welfare organization and community-based mental health care centers. For the second group, family education services, psychosocial rehabilitation, and emergency services can be provided by dialing 110 or 115; (d) Daycare and rehabilitation services. For a population of 100,000 , about 100 people would be eligible for receiving daycare services, which requires the establishment of 2-4 daycare centers so that each center can provide services to 30-50 people; (e) Vocational rehabilitation services: According to various studies, an unemployment rate of $70 \%$ seems reasonable for psychiatric patients. Based on the calculation results, for 750 patients with severe mental diseases, there would be a need for providing vocational rehabilitation service to 52 people, which can be met through supportive/productive workshops and training of business owners, industries, and non-governmental organizations; (f) Accommodation services. In Iran, according to the cultural situation and extended families, the number of homeless psychiatric patients is $1 \%$. In other words, for 750 severe psychiatric patients per 100,000 population, there would be about 8-10 homeless patients. On the other hand, for a typical family of four in Iran, there would be a need for an $80-\mathrm{m}^{2}$ apartment; i.e. per 100,000 population, they can be accommodated in two $80-\mathrm{m}^{2}$ apartments each with a capacity of 4 people.

\section{Discussion and Conclusion}

This study aimed to provide an optimal model of comprehensive urban mental health services and rehabilitation for patients with severe psychiatric diseases, based on collaboration between family physicians and the organizations that provide health care services. Since the current health system allocates a very small amount of budget to psychi- 


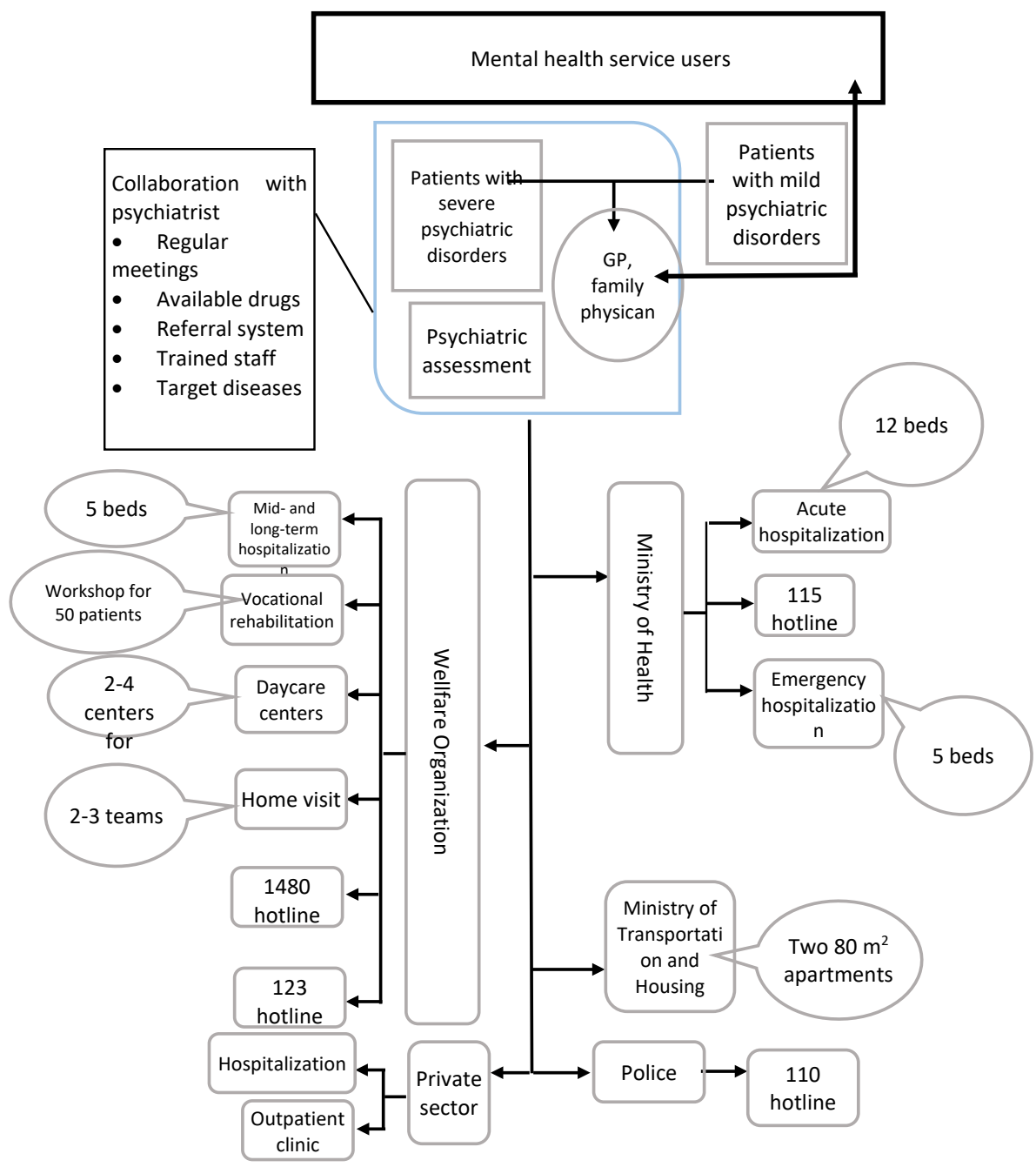

Iranian Journal of
PSYCHIATRY AND CUINICAL PSYCHOLOGY

Figure 1. Inter-organizational cooperation and the contribution of each to provide services to the world of critically ill patients in the urban mental health model

atric hospitals which provide services to acute psychiatric patients for hospitalization, a coherent system can be useful for optimal management of at least one-fifth of the mental health needs of the community. There is a need for coordination between the organizations providing these services to plan carefully, eliminate similar services, and prevent the waste of human capital and resources.

\section{Ethical Considerations}

\section{Compliance with ethical guidelines}

All included studies would be cited in all reports and in all future publications.

\section{Funding}

This project was supported by the Ministry of Health, the Office of Mental Health and Addiction, and the Vice-Chan- cellor for Research and Technology of Shahrekord University of Medical Sciences.

\section{Authors contributions}

All authors equally contributed to preparing this article.

\section{Conflicts of interest}

The authors declared no conflict of interest.

\section{Acknowledgements}

We want to thanks the Mental Health Office Social and addiction Ministry of Health and Medical Education, and the Vice-Chancellor for Research and Technology of Shahrekord University of Medical Sciences. 
مقاله مرورى:

\section{طرح سلامت روان شمرى: توسعه همكارى بينبخشى، نقش محورى يزشك خانواده براى مديريت

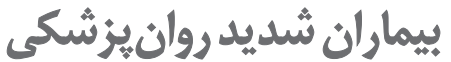

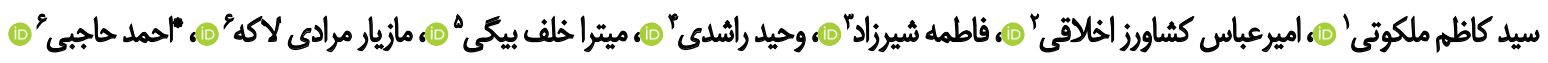
ا. مركز تحقيقات بهداشت روان، دانشكده علوم رفتارى و سلامت روان (انستيتو روانيزشكى تهران)، دانشكاه علوم يزشكى ايران، تهران، ايران.

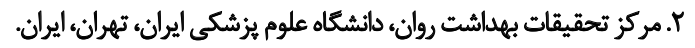

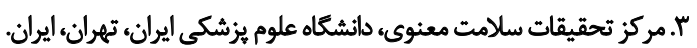

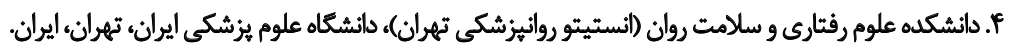

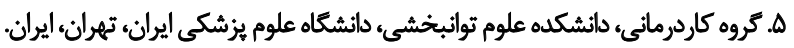

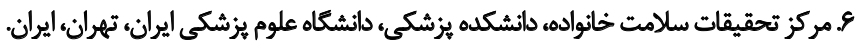

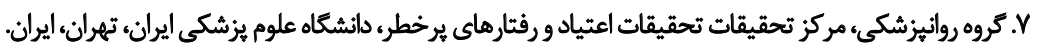

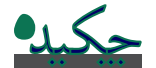

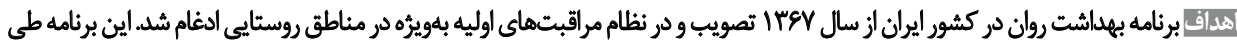

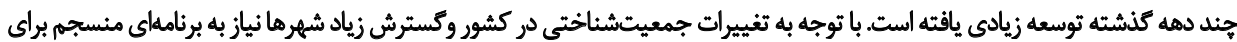

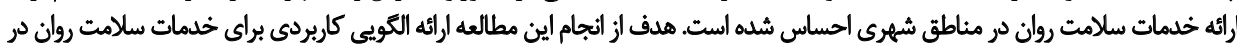

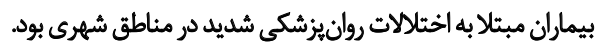

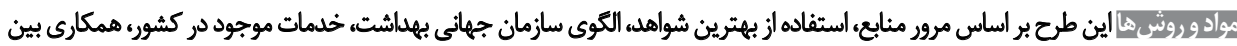

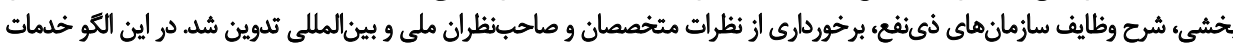

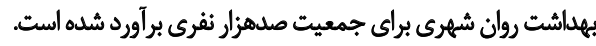

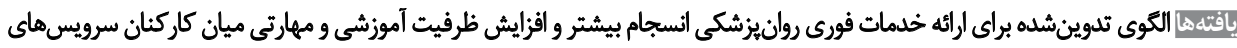

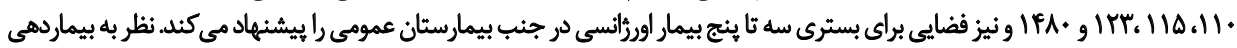

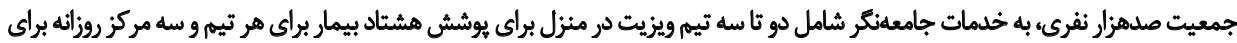

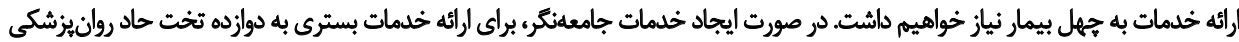

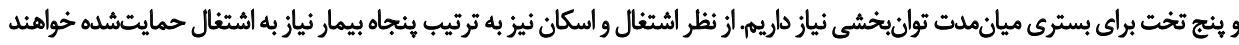

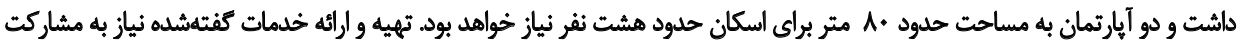

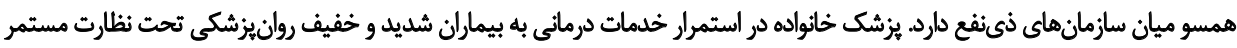

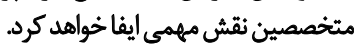

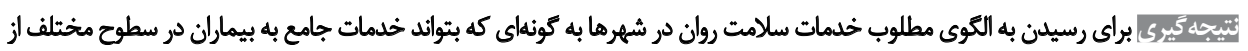

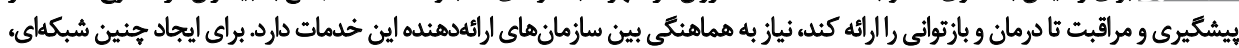

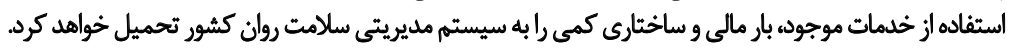

تاريخ دريافت: T Tآدر تاريخ هذيرش: آتخرداد

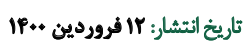

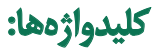
سلامت روان، خدمات

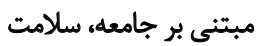

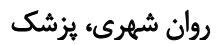
خانواده، خدمات بسترى بريك روانئنشكي

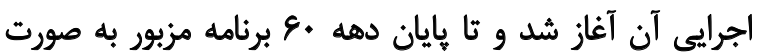

مقامه

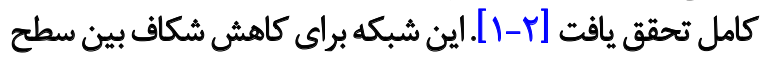

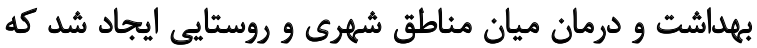

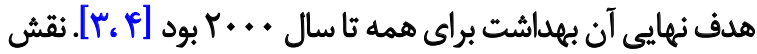

مطالعات مربوط به ايجاد شبكه مراقبت بهداشت اوليه در ايران

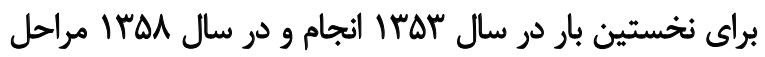

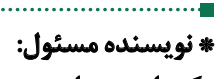

دكتر احمد حاجبي : نوينده مناني نشانى: تهران، دانشكاه علوم يزشكى ايراني، مركز تحقيقات اعتياد و رفتارهاى يرخطر، كروه روانيزشكى.

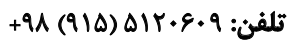
hajebi.a@iums.ac.ir يست الكثرونيكي 
شهرى در كشور در تزارش سال هAr إ سازمان جهانى بهداشت

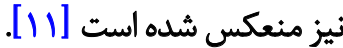

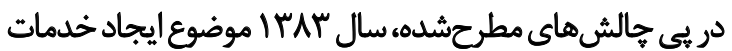

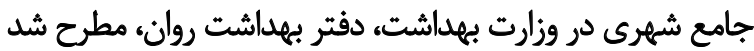

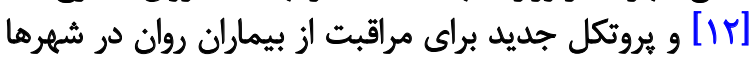

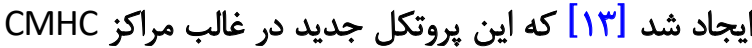

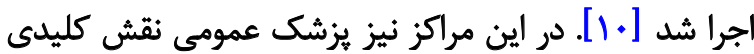

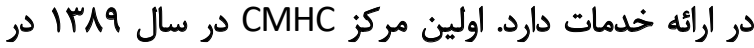

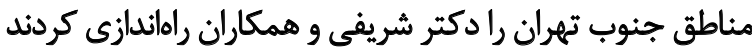

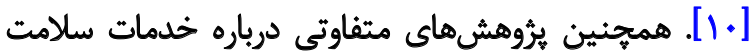

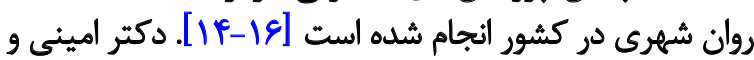

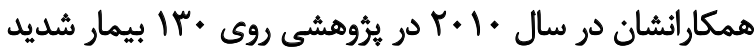

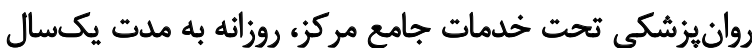

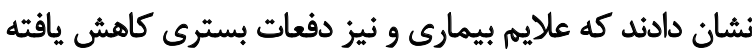

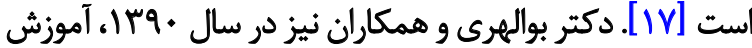

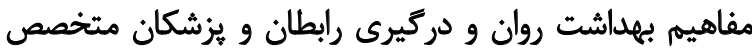

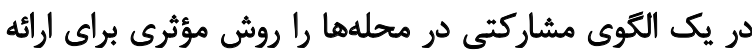

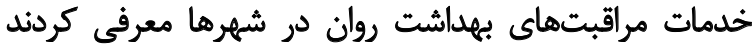

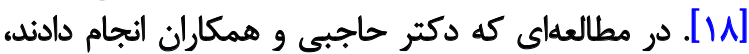

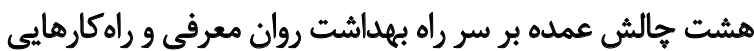

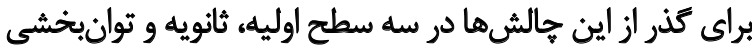

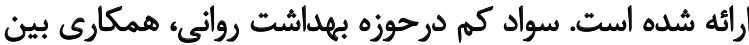
بخشى ضعيف، كمبود خدمات، كيفيت نامطلوب در در ارائه خدمات برات

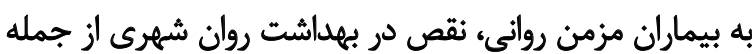

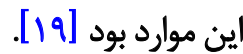
براى رهيافت به يك برنامه جامع بهداشت روان شهرى، دفتر

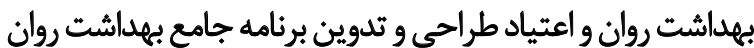

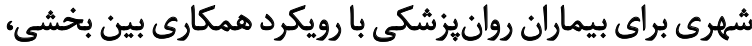

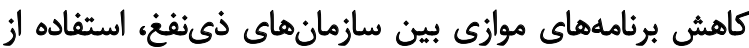

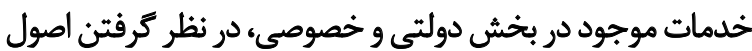

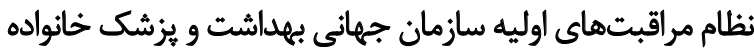

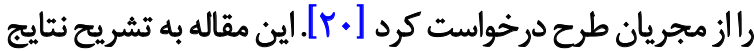

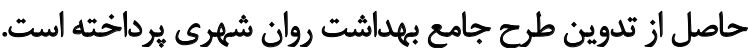

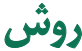

اين مطالعه با روش مطالعه كتابخانهاي، بررسى وضعيت موجود' و استفاده از نظرات متخصصان بَ انجام شد.

روش اجرا: براى تدوين طرح بهداشت روان شهرى اقداماتى

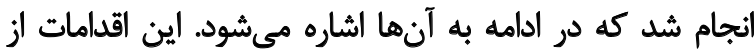

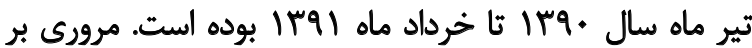

1. Situational analysis

2. Expert opinion
محورى ارائه خحمات در اين سيستم بر عهله يزشكان عمومى بودا.

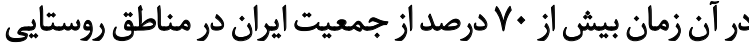

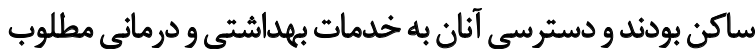

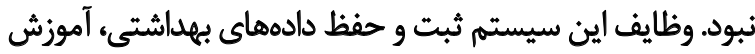

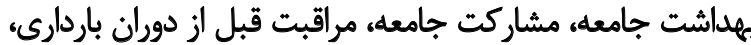

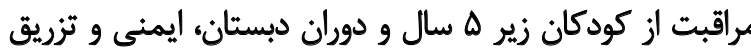

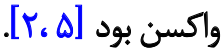

در اين سيستهه اولين سطح خدمات برعهده بهات بهورزان خانه

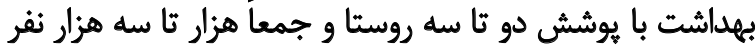

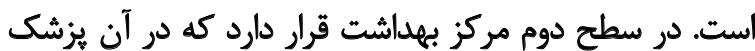

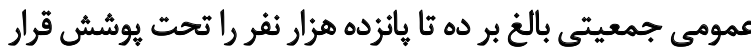

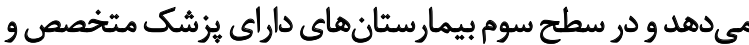

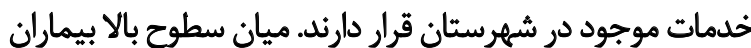

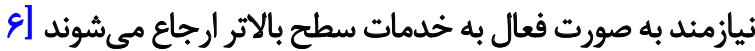

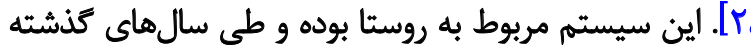

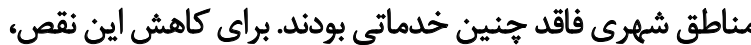

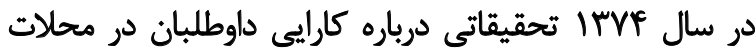

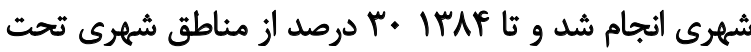

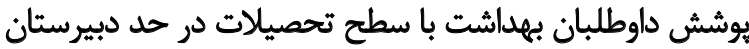

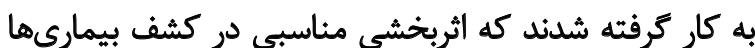

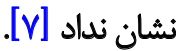

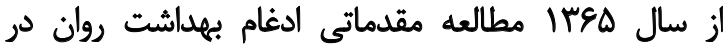

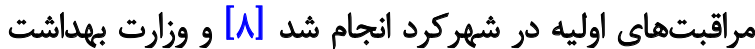

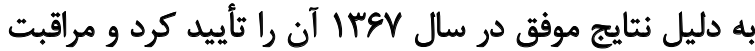

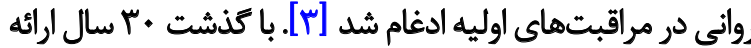

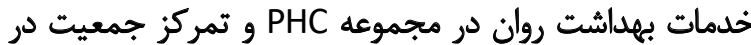
مناطق شهرى اثربخشى آن را با ابهام و ترديد مواجه كرئ كرده است

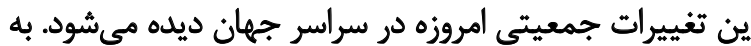

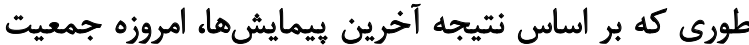

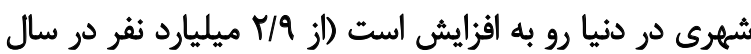
رش ب...

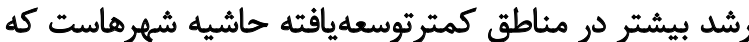

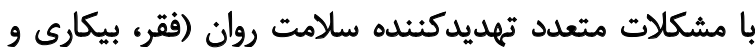

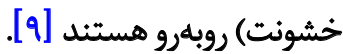
كشور مانيز با تغييرات مهم جمعيتى و شهرنشينى مواجه شده

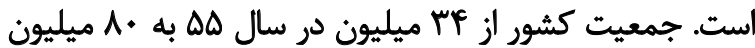

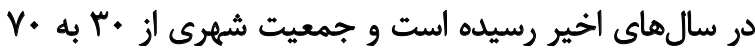

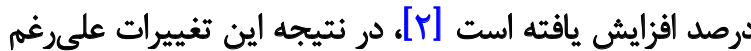

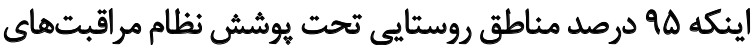

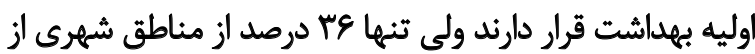

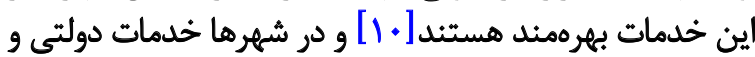

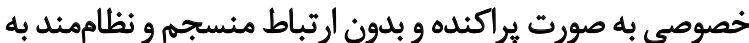

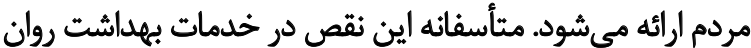


نسخه اول در يانل تخصصى با حضور متخصصان صاحبنظر

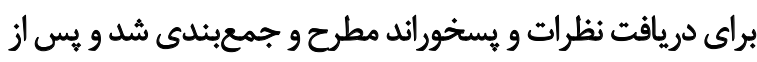
اصلاح نظرات نسخه نهايى به دست آمد.

هافتهاها

براى جمعيت صدهزارنفرى نياز به خدمات اوررانس

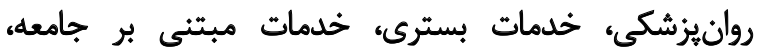

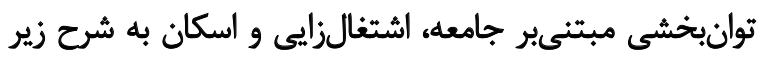

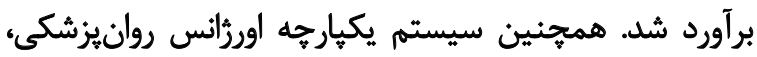

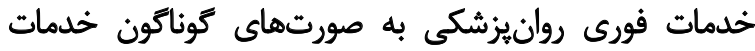

$$
\text { تلفنى، تيمهاى سيار و بخش بسترى ارائه مى بـودي }
$$

$$
\text { مُط تلفنى بحران }
$$

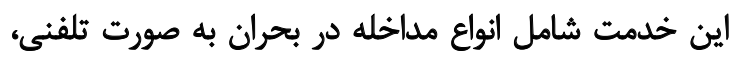

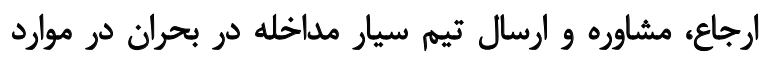

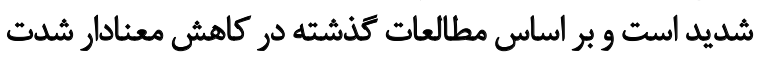

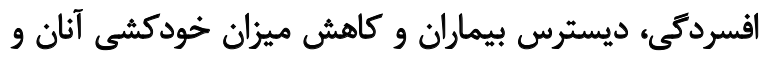

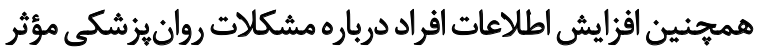

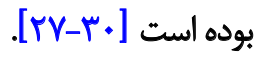

$$
\text { تيمرهاى سيار مداخله در بحران }
$$

جمعبندى نتايج نشان داد كه استفاده از اين خدمث بستر بادرى

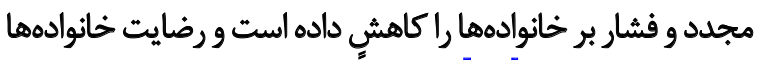

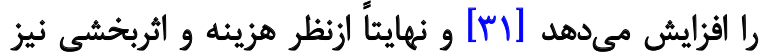

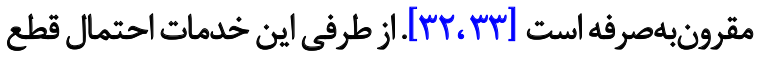

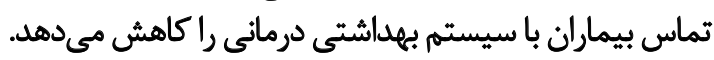

در جمعبندى نهايى اين طرح با توجه به اينكه در كشور ما

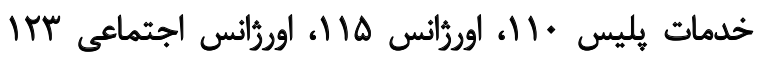

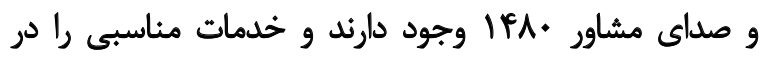

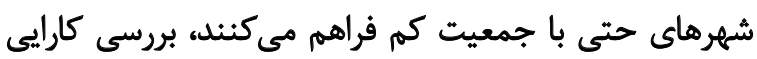

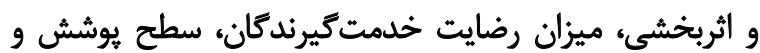

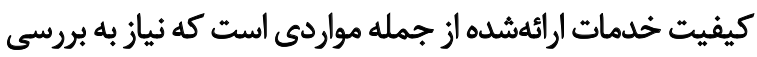

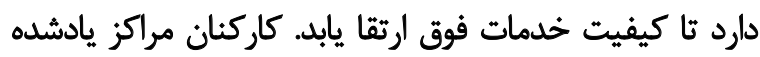

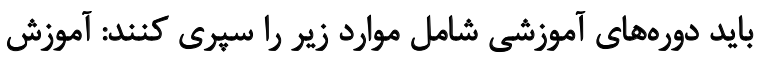

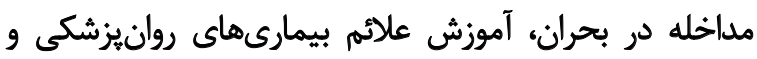

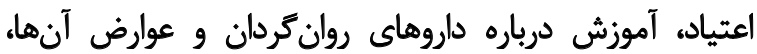

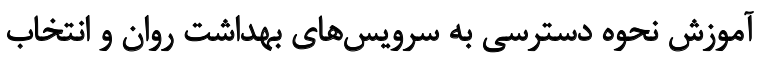

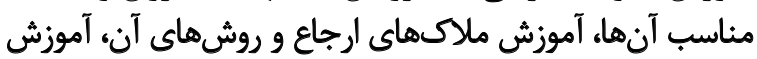
توجه به اختلافات فرهنكى و آموزش آشنائى باقوائين.

$$
\text { خدمات فورى ووان بيزشكى ميثئير بيماروسثان }
$$

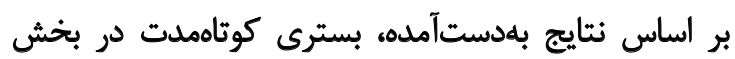

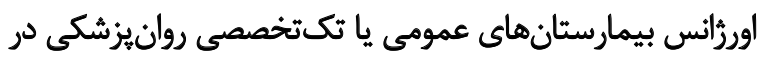

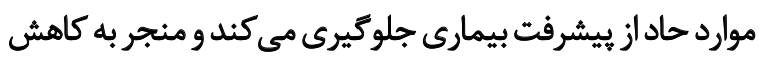

مقالات و نتايج آخرين بيمايشهاى سلامت روان در ايران و بار

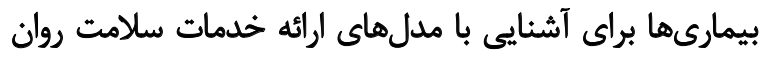

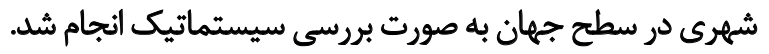

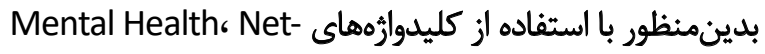
work Urban Mental Health و Community Mental Health

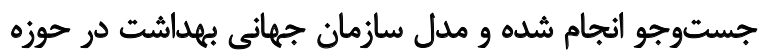
مراقبت سلامت روان نيز ارزيابى شد مدان

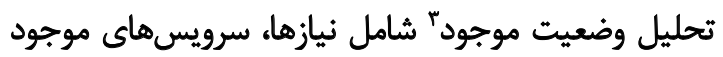

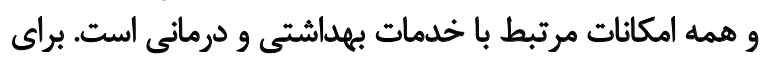

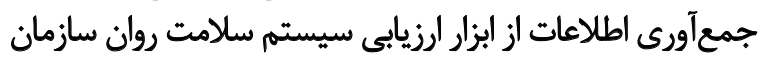

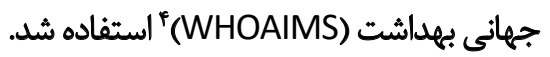

$$
\text { مرور اسئاد بالادستى ملى و بين المللى بهانى }
$$

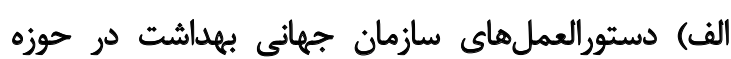

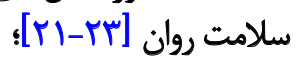

ب) دستورالعمل خدمات مراقبت اوليه مبتنىبر هزشكى روني

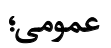

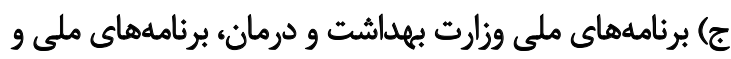

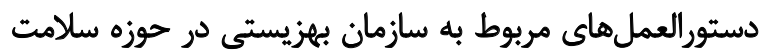

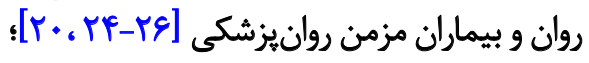

بهرهمندى از نظرات مشورتى متخصصان بين المللى، شامل: 1.

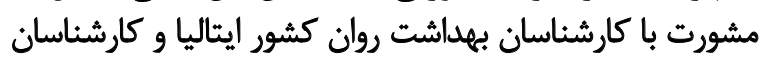

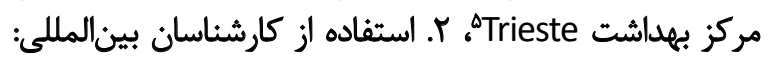

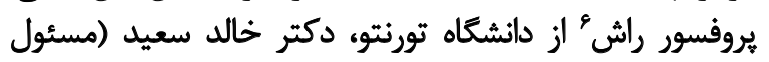

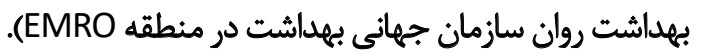
تشكيل تيمهاي تخصصى براي مطالعه و تدوين بخشهاي

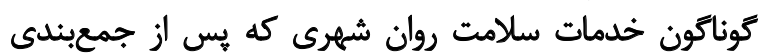
مطالعات و نظرات متخصصين به دست آمدات سلامد

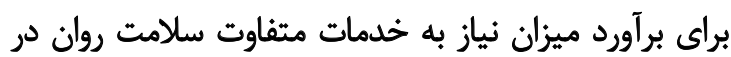

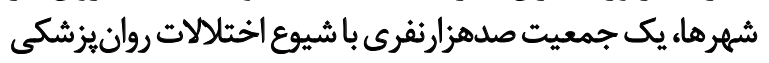

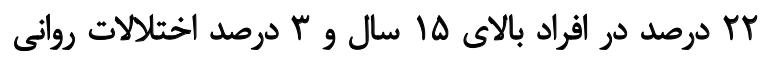

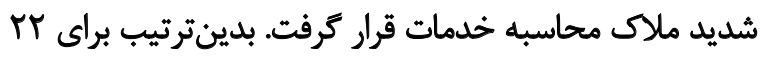

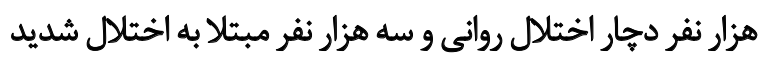
و مزمن روانى خدمات محاسبه و طراحى شد.

3. Situational analysis

4. World Health Organization-Assess important component of the mental health(WHO-AIMS)

5. WHO Collaborating Centre for Research and Training in Mental Health, Trieste (Italy)

6. Rush 
جدول ا. تعداد بيماران مد نظر بر اساس آمار بيماران بالاى ها سال (ه هزار نفر)

\begin{tabular}{|c|c|c|c|c|}
\hline تعداد مدنظر موارد شديد & درصد مدنظر موارد شديد & تعداد كل مدنظر در جمعيت & شيوع (درصد) & اختنالات \\
\hline rva & $1 .$. & TVA & .10 & اسكيزوفرنيا \\
\hline Tro & $1 .$. & TVA &.$/ 0$ & اختلال دوقطبى \\
\hline va. & $r$. & rVa. & $\Delta$ & اختالال افسردكى عمده \\
\hline 10 . & r. & vo. & 1 & 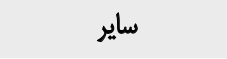 \\
\hline $1 \% \Delta$. & - & DTS. & 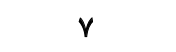 & جمع \\
\hline
\end{tabular}

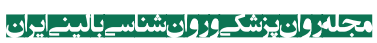

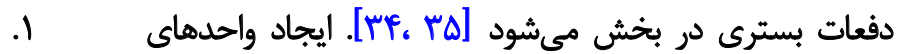

$\frac{100000 \times 1.03}{1000}=103$

(تعداد بسترى در مدت يك سال براى جمعيت صدهزارنفرى)

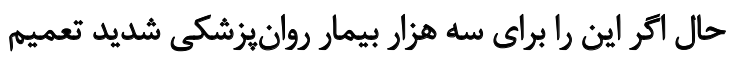

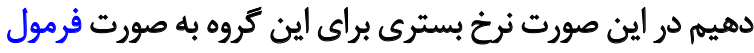

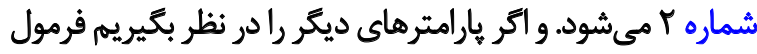

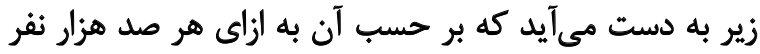
جمعيت V/D تخت براى بيماران روانيز بـ ديكى حاد نياز خواهيم داشت. - اشعيت

$$
\frac{103 \times 100}{3000}=3.5 \%
$$

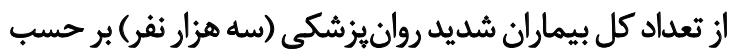

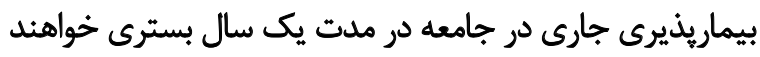

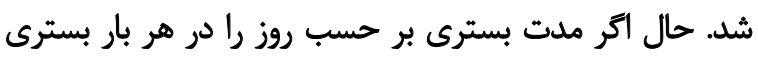

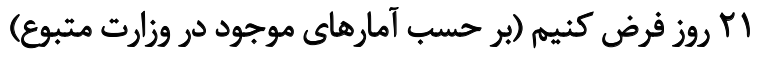

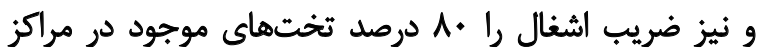

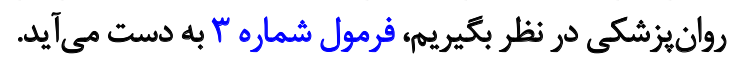

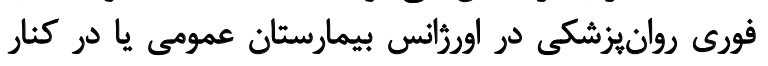

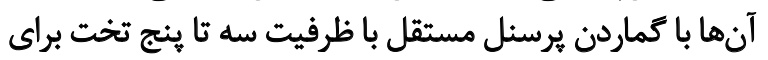

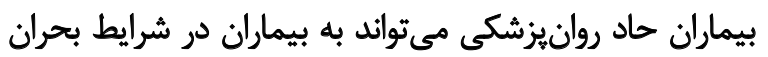

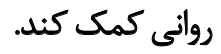

$$
\text { خدمات بسترى كوثاه و طولاتيىمدث }
$$

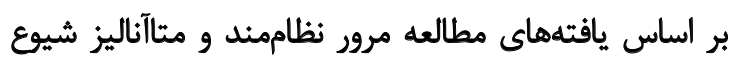

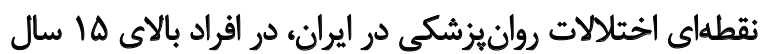

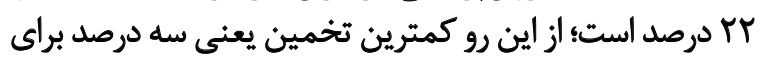

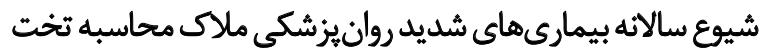

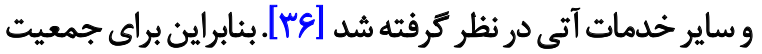

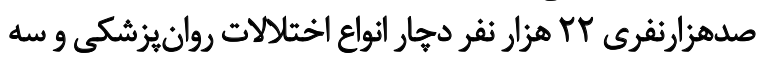

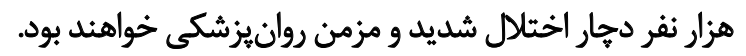

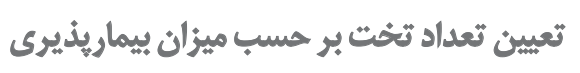

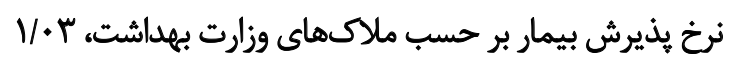
به ازاى يك هزار نفر جمعيت در يك سال است (فرمول شماره

\begin{tabular}{|c|c|c|c|c|}
\hline 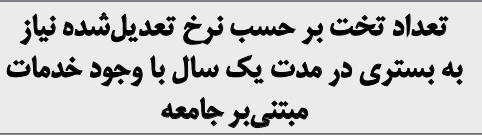 & خدمات مبتنى تخد بداد تخعه & طول متوسط بسترى & درصد نياز براي بسترى & اختلال \\
\hline$(\Delta) \%$. & ir & m & $\Delta$. & اسكيزوفرنيا \\
\hline$(\Delta) \%$. & ir & $m$ & $\Delta$. & اختلال دوقطبى \\
\hline (1) $\%$ & $r / \Delta$ & $M$ & $\Delta$ & اختّلال افسردكى عمله \\
\hline$(N / T) \%, r$ & r & r & r. & ساير \\
\hline $1 \pi / r$ & $r . / \Delta$ & & & جمع \\
\hline
\end{tabular}

جدول r. تختهاى مورد نياز براى مراقبت روانيزشكى حاد 


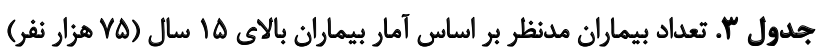

\begin{tabular}{|c|c|c|c|c|c|c|c|}
\hline & تهد خدماتى از بيماران كه نياز & 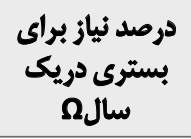 & تعداد مدنظر & مورارد شديد مدنظر & ثرداد كل مدنظر & شيوع(درصد) & اختلالات \\
\hline & $11 \%$ & $r$. & MVD & 10 & mo &.$/ \Delta$ & أسكيزوفْرنيا \\
\hline & $11 \%$ & $r$. & rVa & $1 \ldots$ & rva &.$/ \Delta$ & اختلال دوقطبى \\
\hline & r & r & Vo. & $r$. & rva. & $\Delta$ & اختّلال افسردكى عمله \\
\hline & 10 & 1. & 10. & r. & $V \Delta+$ & 1 & ساير \\
\hline$\%$ & (8) & & 180. & - & $\Delta r \Delta$. & $v$ & جمع \\
\hline
\end{tabular}

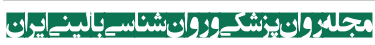

طول سال در دسترس باشد. فرمول محاسبه تخت براى بيماران

.$r$

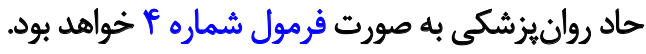

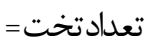

تعدادمواردشديد×نيازبهبسترى(./) ×فاكتور :رخش

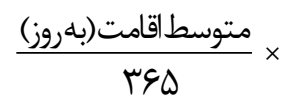

بر اساس يافتههاي مطالعات انجامشده در ايران با فرض نبود

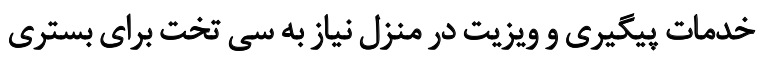

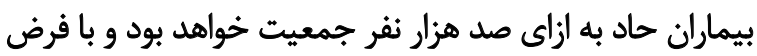

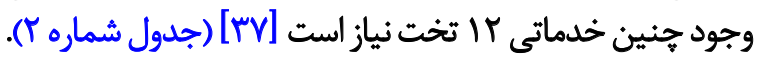

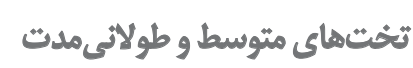

فرض بر اين است كه در يك شهر صدهزارنفرى با وجود حمايتهاي

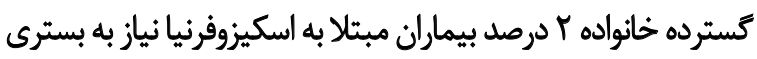

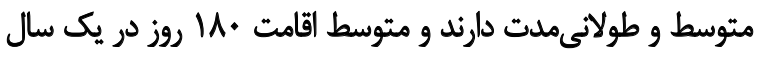

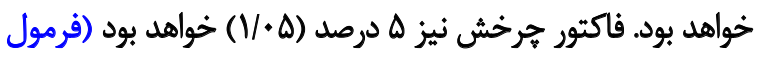

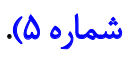

$$
\begin{aligned}
& \text { تعدادتخت = دنا: } \\
& \text { تعدادمواردشديد ×نيازبهبسترى(./) ×فاكتور جرخش }
\end{aligned}
$$

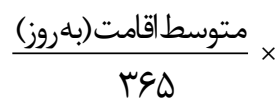

$$
\begin{aligned}
& =375 \times 2 \% \times \frac{18.85}{365}
\end{aligned}
$$

درصورتى كه روند كاهش نياز به تخت با وجود خدمات

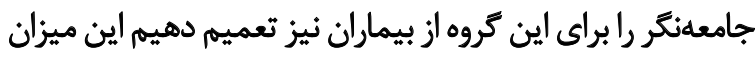
حدود ها تخت كاهش مى ئر رابد.

به طور خلاصه: براى جمعيت صدهزارنفرى در صورت وجود

$$
\begin{aligned}
& \text { تعداد بيمار در جامعه × درصد ضريب بسترى (روى بـري }
\end{aligned}
$$

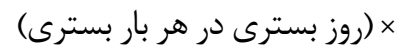

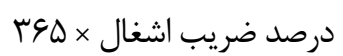

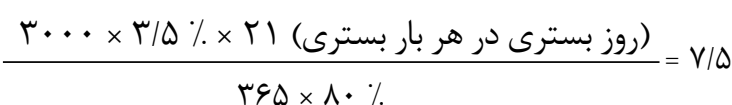

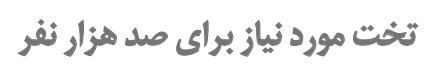

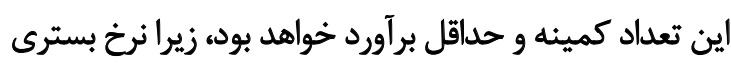

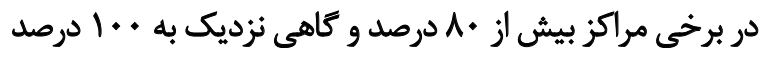

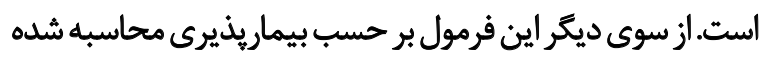

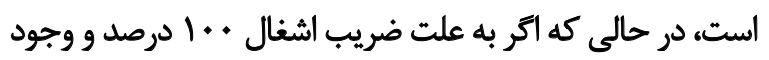

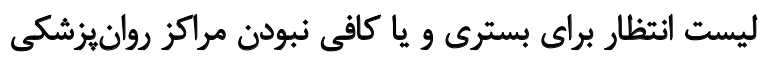

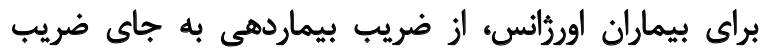

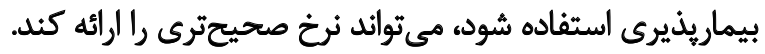

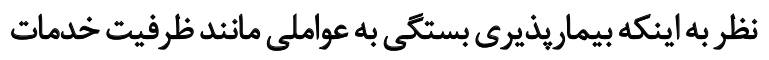

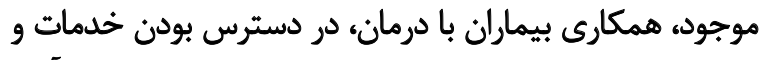

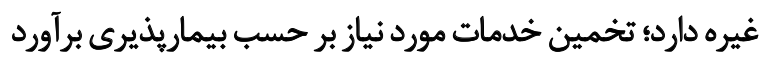
صحيحي رالرائه نخواهد دادي تخدين خدات

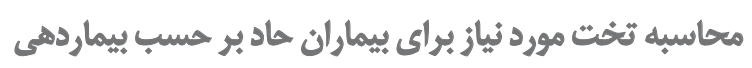
فرض بر اين است كه در يك جامعه با صد هزار نفر، Vه هزار

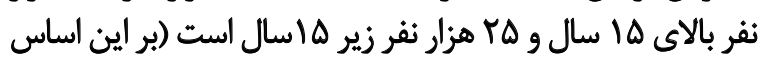

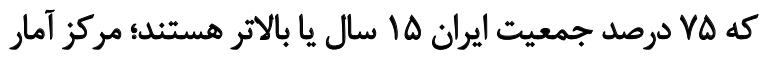

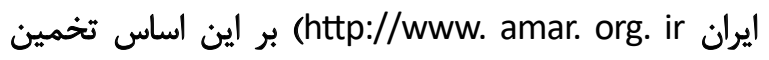

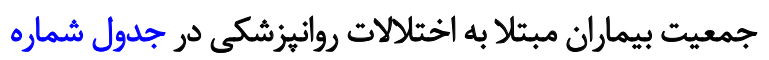
آمده است.

بدينترتيب در منطقهاى باصد هزار نفر خدمات بسترى براى

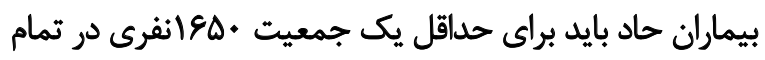




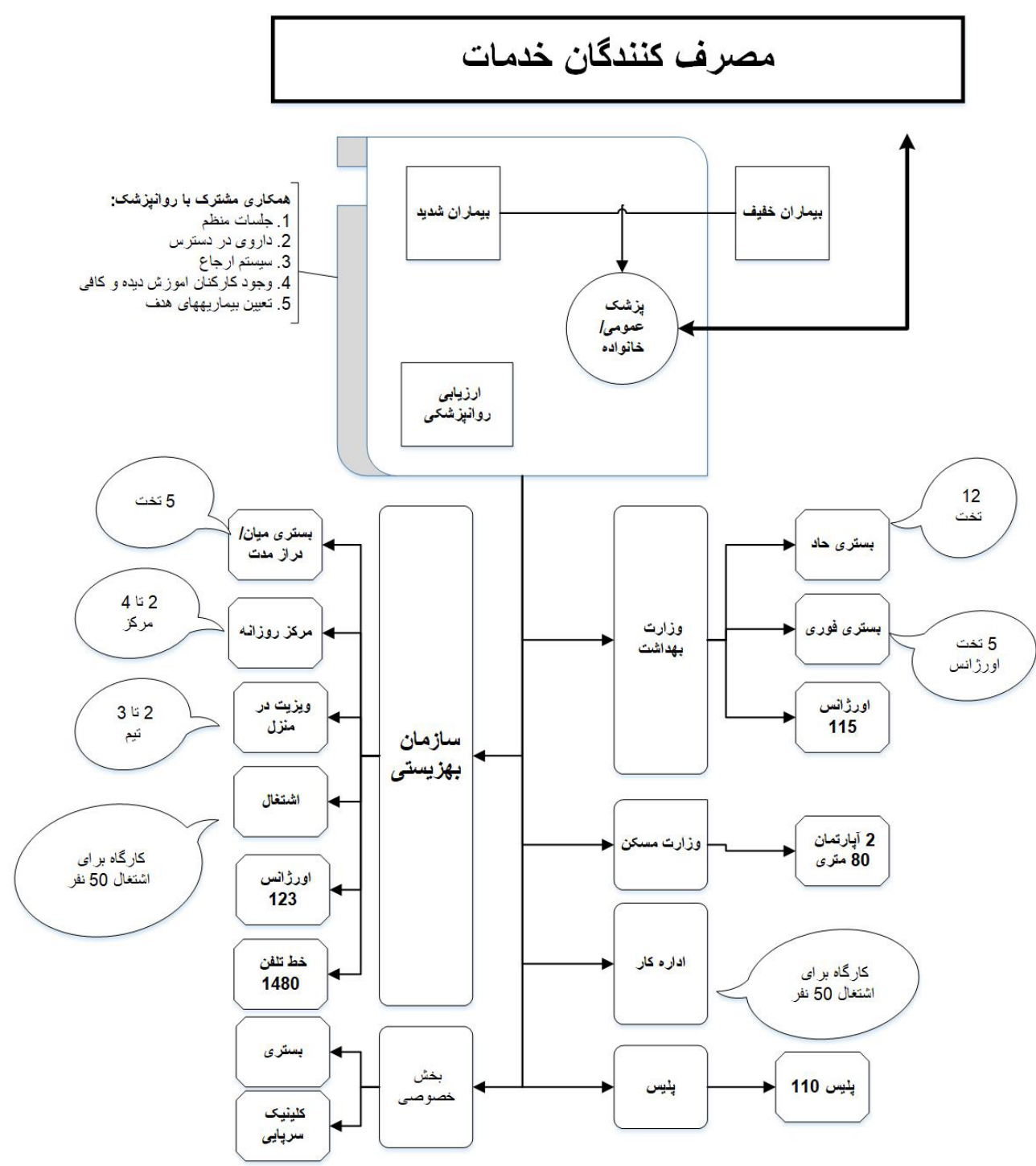

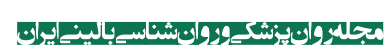

تصوير ا. همكارى بينسازماني و سهم هريك براى تأمين خدمات موردنياز بيماران شديد روانيزشكى در مدل بهداشت روان شهرى

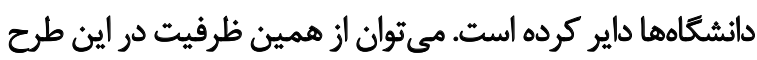

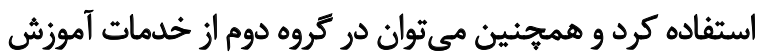

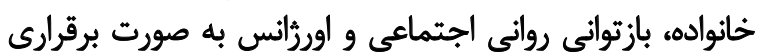

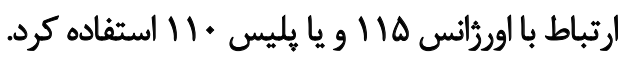

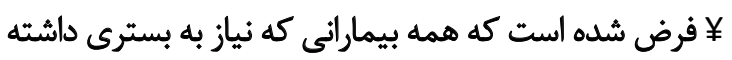
باشند، نياز به خدمات ويزيت در منزل هيمل نيز داشته باشند.

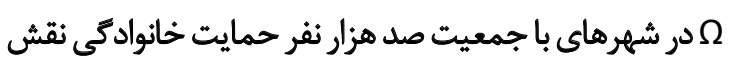

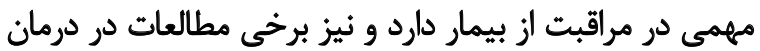

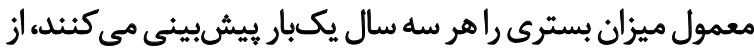

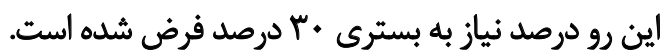

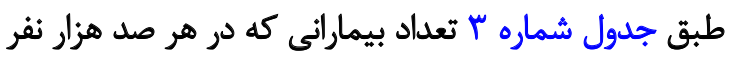

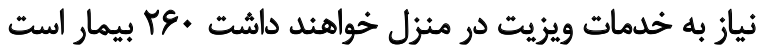

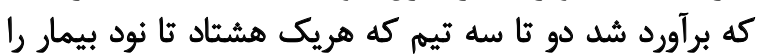

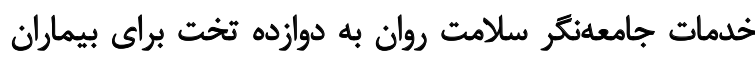

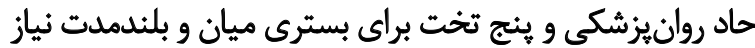

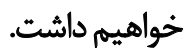
خدمات ويزيت در مثزل

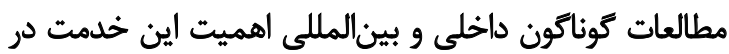

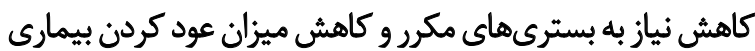

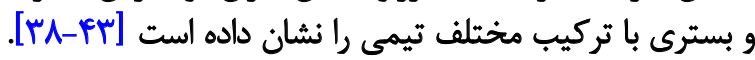

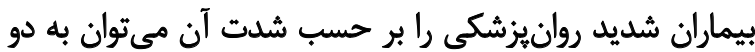

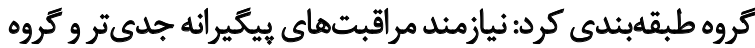

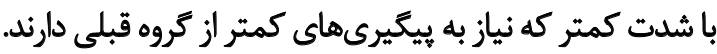
براي كروه اول در حال حاضر خدمات ويزيت در منزل از سوى ني

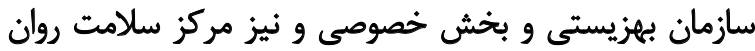

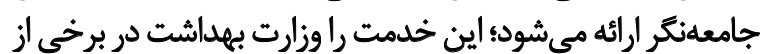




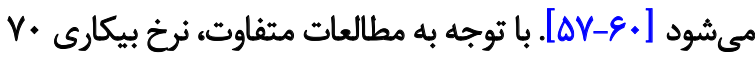

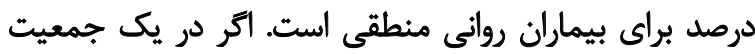
صدهزارنفرى •VD بيمار شديد رواني بانى (اسكيزوفرنيا و اختلال

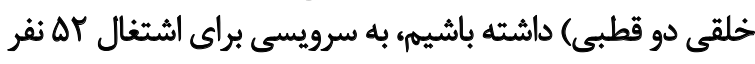

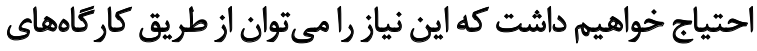

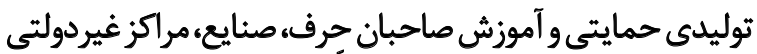

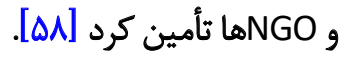

\section{حُدمات اسكان بيماران مزمن روانيزوشكى}

الكوهاى متفاوتى براى اسكان اين بيماران از اقامت اجبارى تأيات

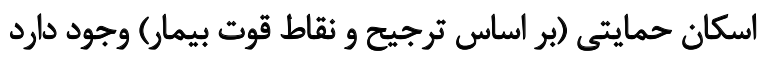

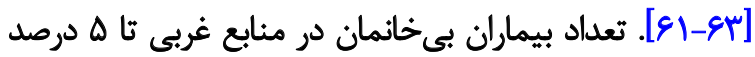

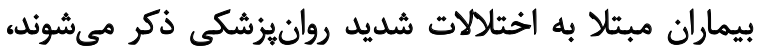

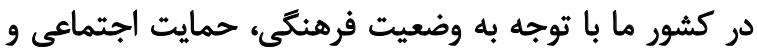

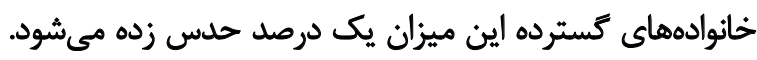

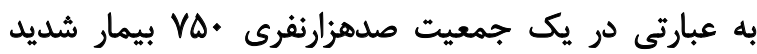

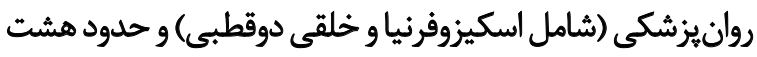

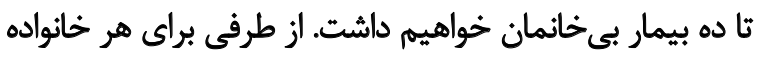

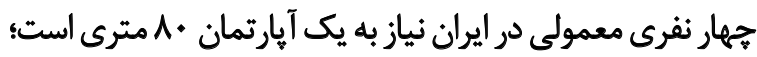

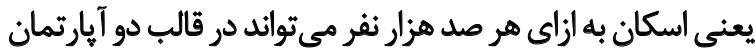

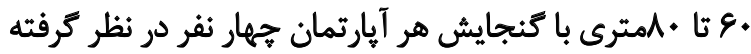

شود.

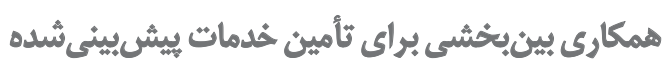

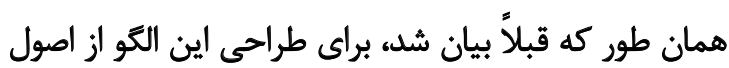

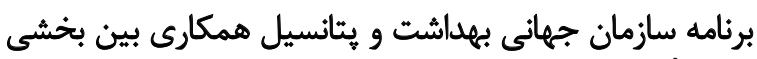

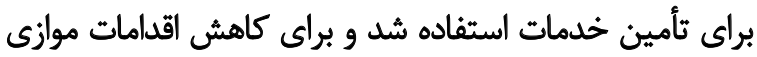

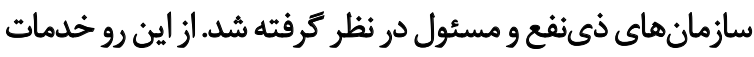

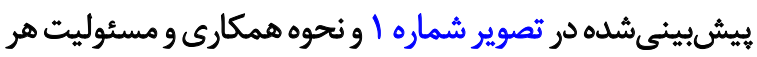

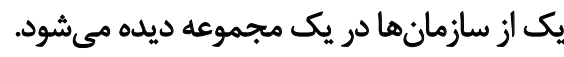

بحث

هدف از تدوين ״الكوى جامع بهداشت روان شهرى"

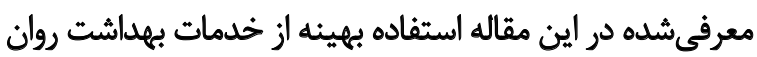

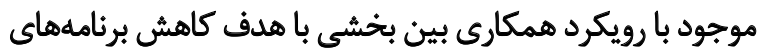

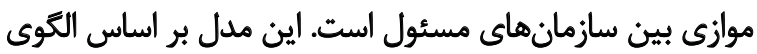

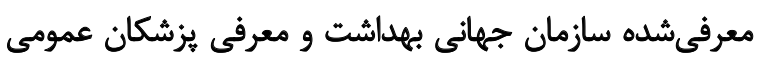

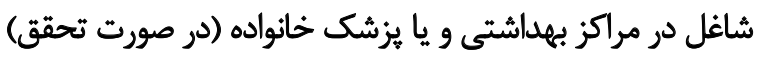

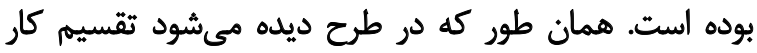

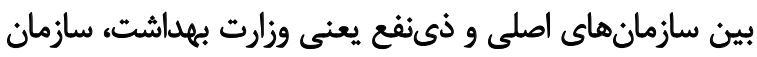

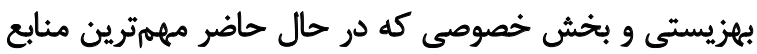

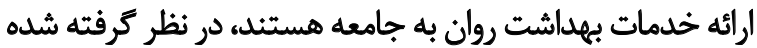

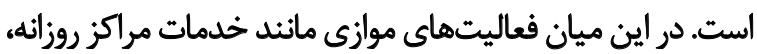

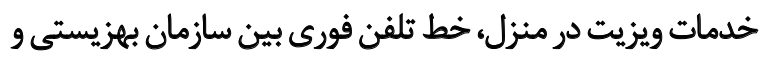

تحت يوشش قرار دهند، مى تواند به نياز اين جمعيت باسخ دهد إندا

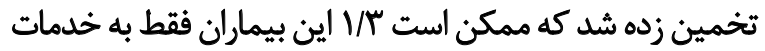

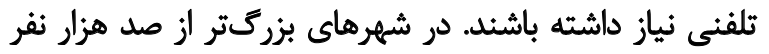
مى توان براى يكسوم بيماران تيم بيكيرى تلفنى در نظر ترفت.

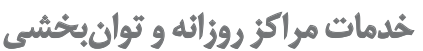

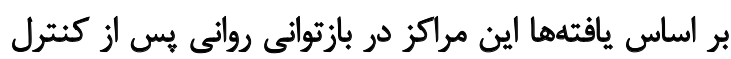

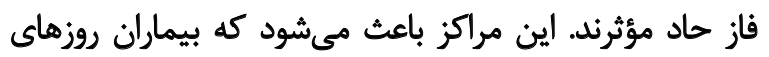

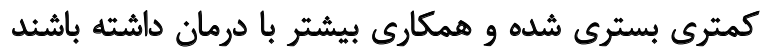

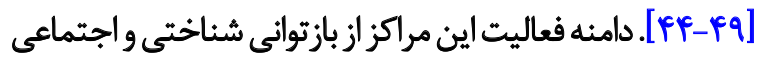
تا بازتوانى هاى شغلى كسترده است.

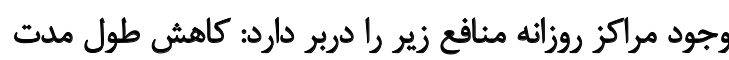

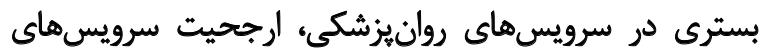

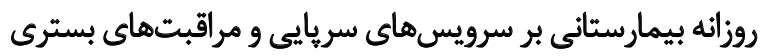

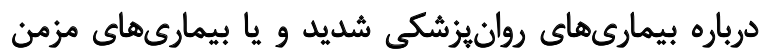

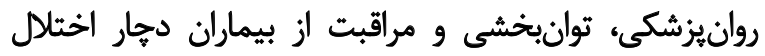

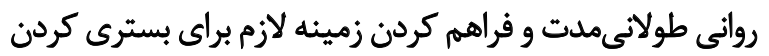

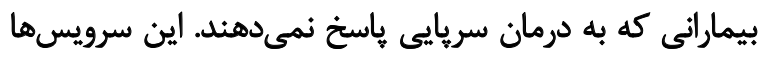

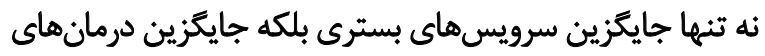
سريايي هستئد. تئين

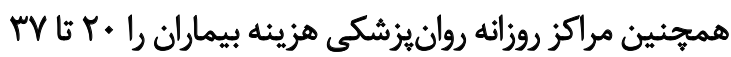

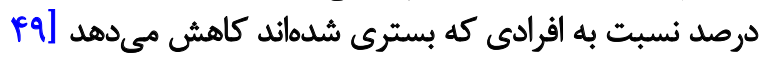

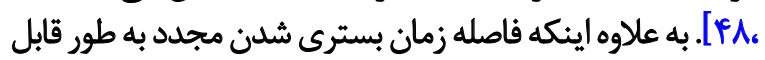

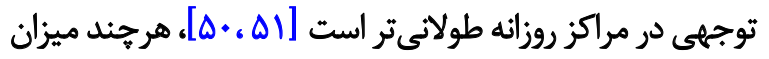

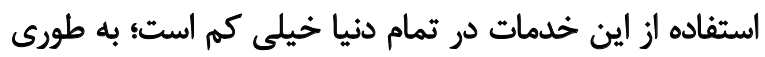

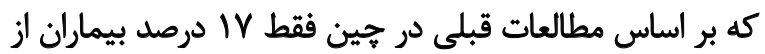

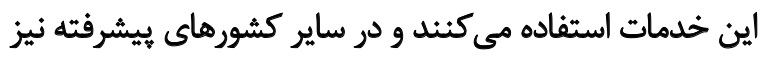

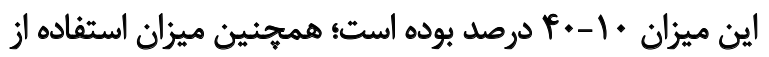

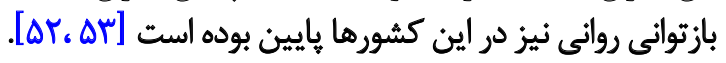

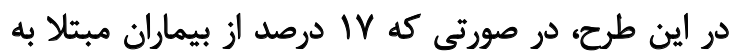

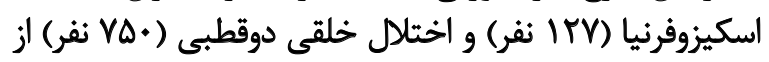

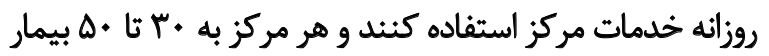

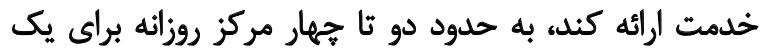
جمعيت صدهزارنفرى نياز خواهيم داشت ائه

\section{حلدمات بإزتوائى شغلي}

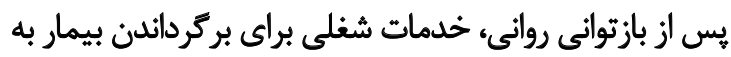

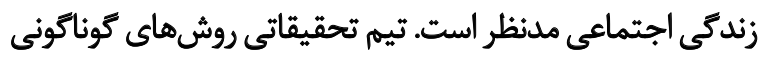

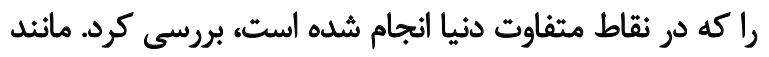

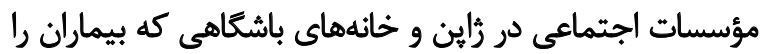

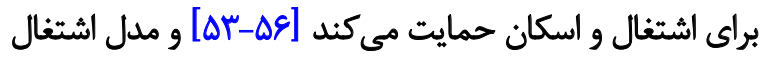

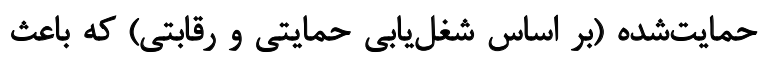
افزايش اشتغال و رضايت بيماران و افزايش حمايتى كيفيت زندئى آنائن 
روانى شامل روانشناس و روانيزشك شاغل در مراكز بهداشتى

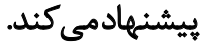

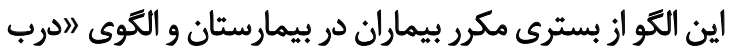

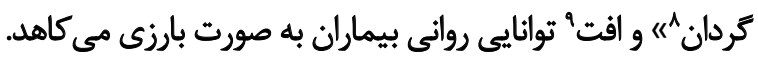

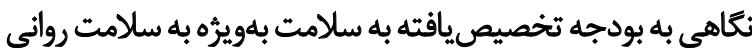

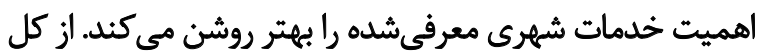

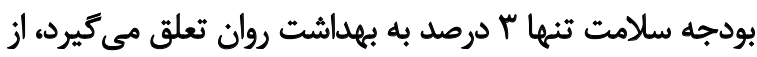

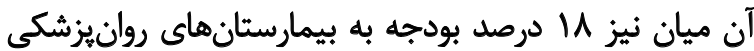

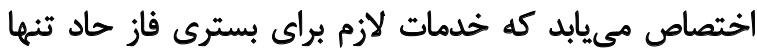

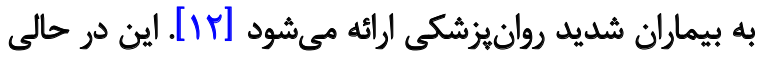

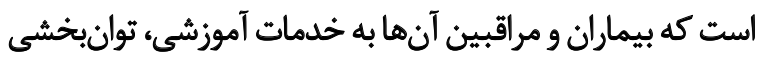

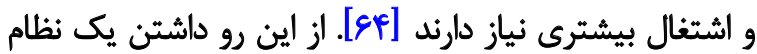

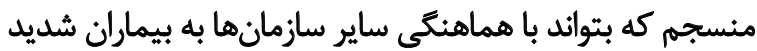

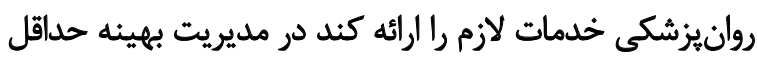

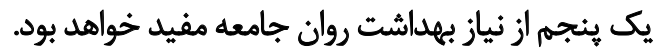

يس إز إيان تدوين ״الكوى بهداشت روان شهرى" اجرا يايلوت

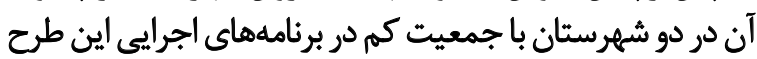

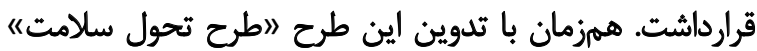

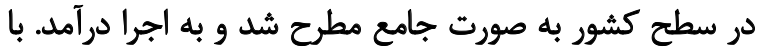

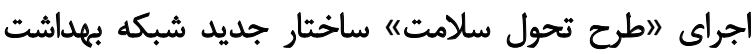

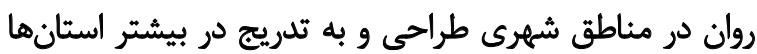

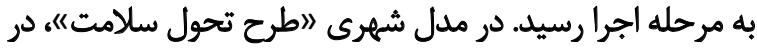

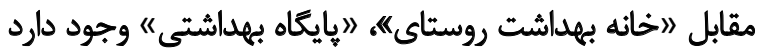

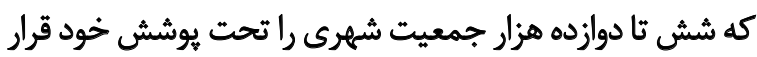

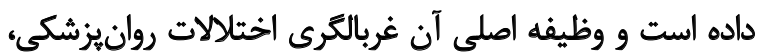

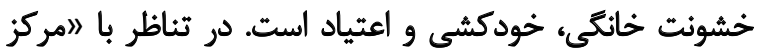

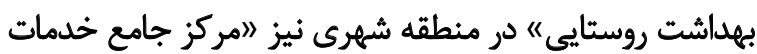

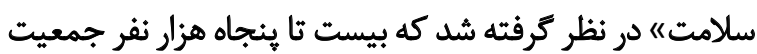

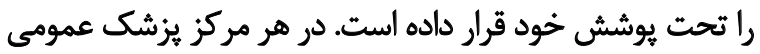

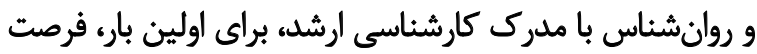

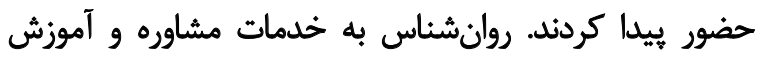

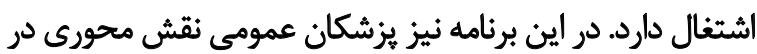

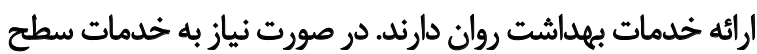

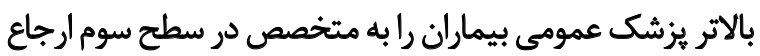

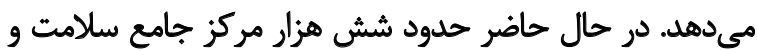
مايكاه شهرى در كشور داير شده است.

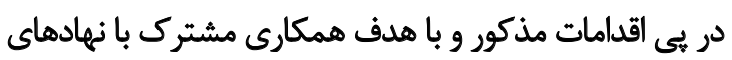

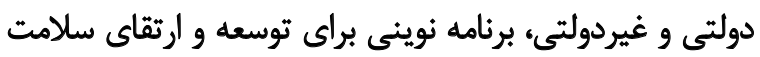

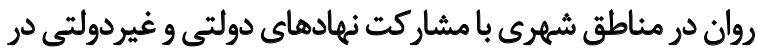

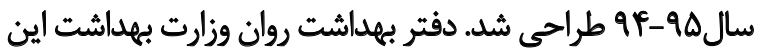

8. Revolving door

9. Downward drift

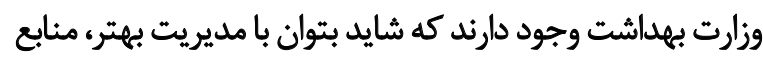

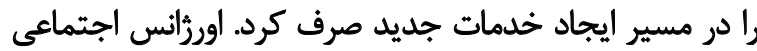

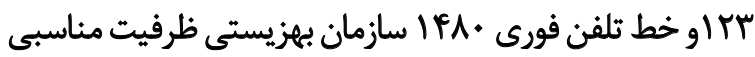

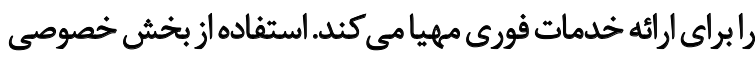

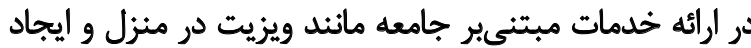

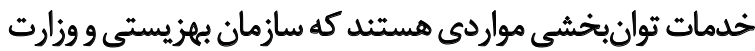

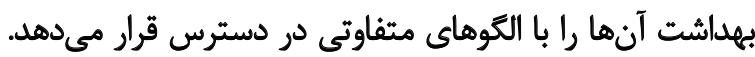

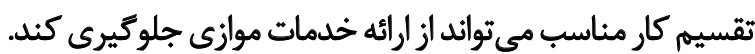

ايجاد مراكز بسترى اوررانس در بيمارستانهاى روانيزشكى و

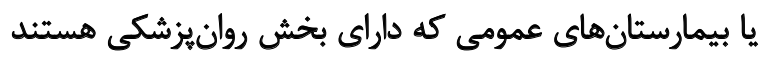

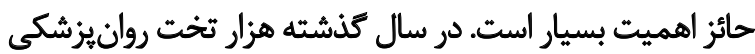

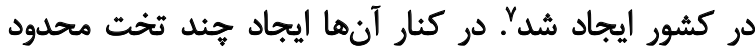

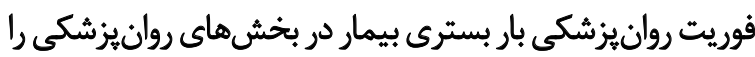

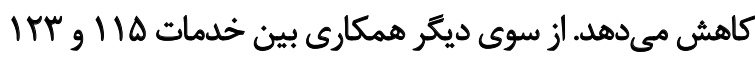

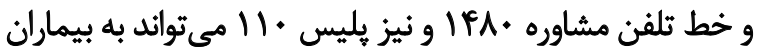

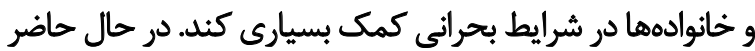

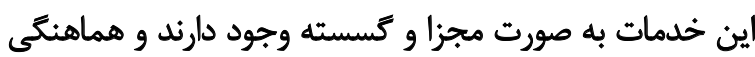

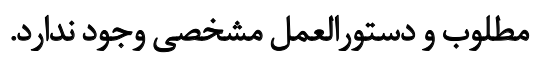

ماهيت بيمارىهاى شديد روانيزشكى به كونهاي است كه

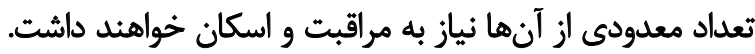

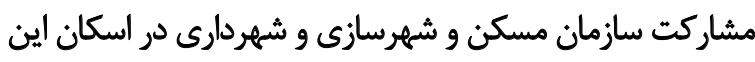

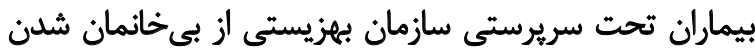
آنها جلو تيرى مى كند.

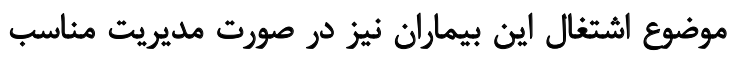

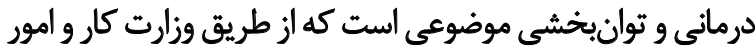

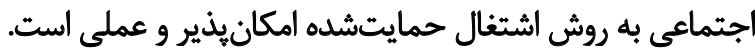

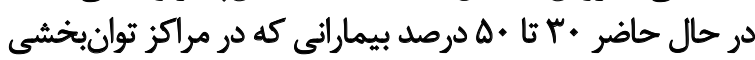

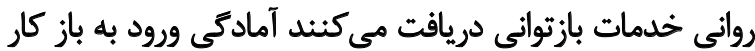

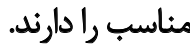

نقش يزشك عمومى به منزله يزشك خانواده در ادرامه درمان

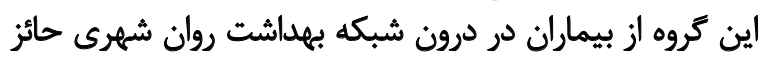

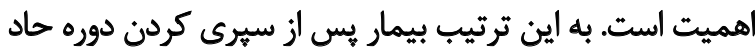

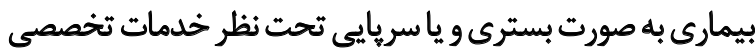

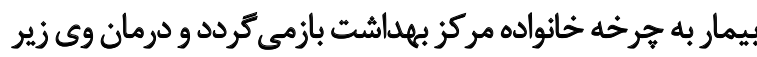

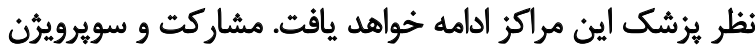

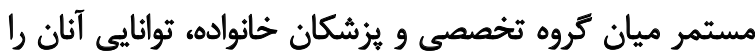

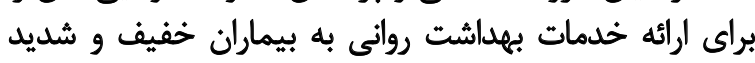

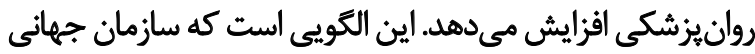

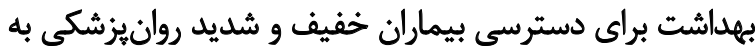

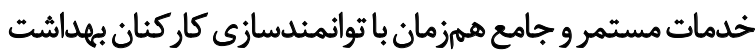

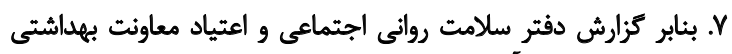

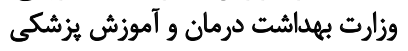


است، بيشنهاد مى شود دو طرح لاتحول سلامته و طرح المركز

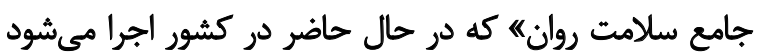

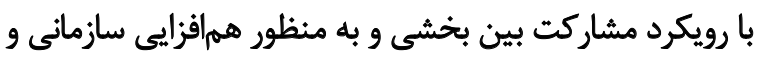
كاهش كارهاى موازى و صرفهجويى در هزينها بارئ بازبينى شوند.

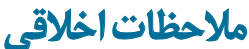$$
\text { بيروى از اصول الخالاق يؤوهش }
$$

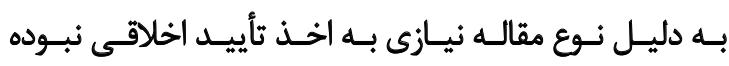

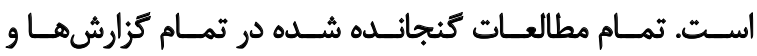

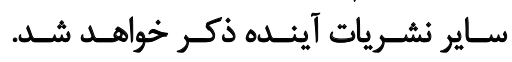

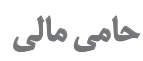

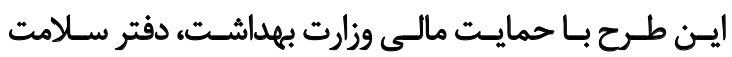

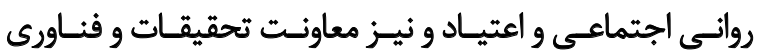

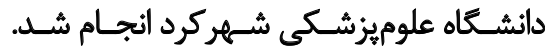

$$
\text { مشاركت نويسند تكان }
$$

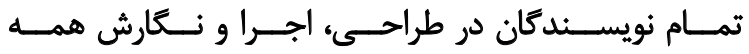

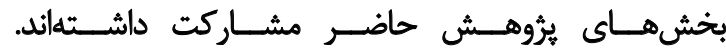

$$
\text { تعارض مثأقع }
$$

بنابر اظهار نويسندكان اين مقاله تعارض منافع ندارد.

$$
\text { تشكر و قدروانى }
$$

بدينوسيله از همكارى وزارت بهداشت، دفتر سلامت روانى

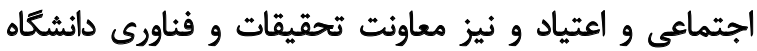
علوميزشكى شهركرد تشكر و قدردانى مي كنيم.
طرح را به نام "طرح خدمات جامع سلامت روانى و مراقبتهاي

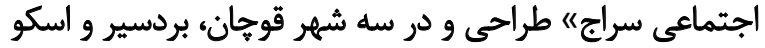

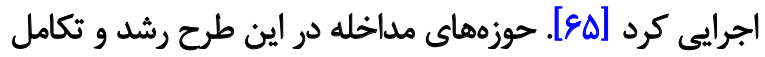

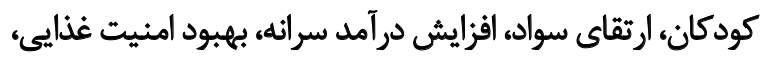

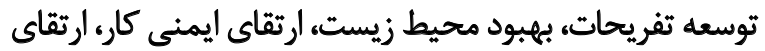

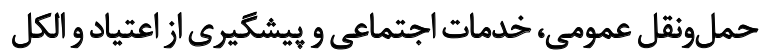

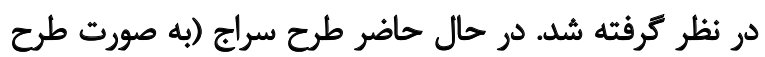
آزمايشى) در سطح كشور در حال اجراست.

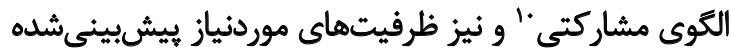

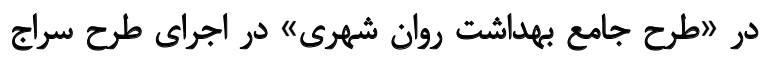
موفقيت بيشترى را براى تحقق بهداشت روان شهرى درى اجراى به همرح سراه

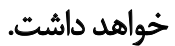

نكاهى به فرايند تحولات و ارتقاى ايجاد شبكه بهداشت

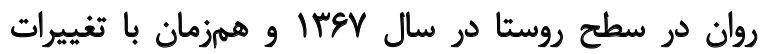

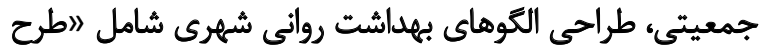

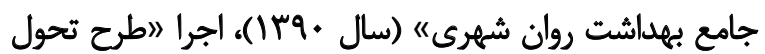

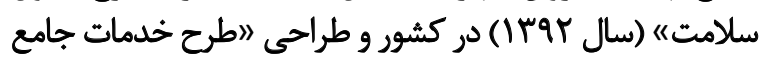

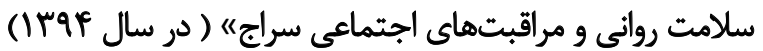

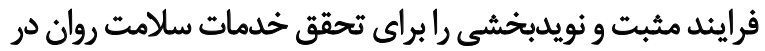
شهر و روستا داشته است. تثيجليرى طى •r سال كذشته مديريت سلامث روان كشور در سطح

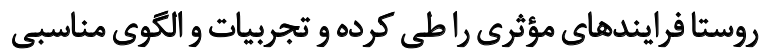

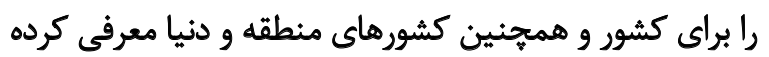

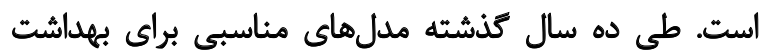

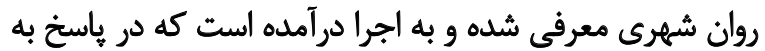

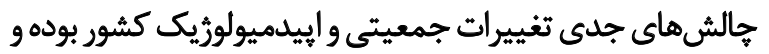

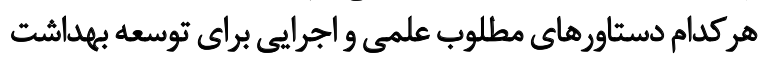

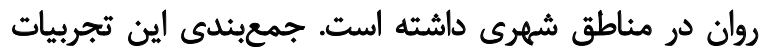

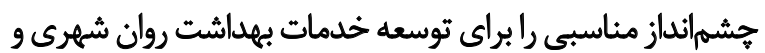
روستايى ترسيم مى كند.

در اين مطالعه به دليل ضيق وقت، دشوارى در دسترسى به به بهان

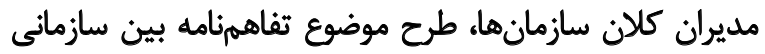

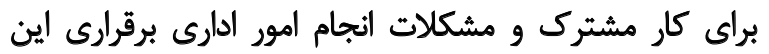

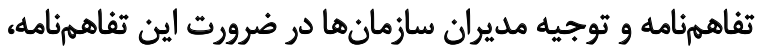

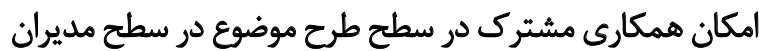

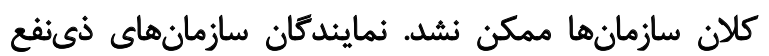

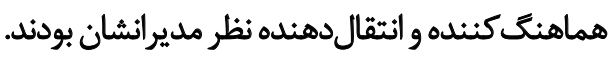
براى مشاركت حداكثرى بين سازمانى كه هدف اصلى اين طرح 


\section{References}

[1] Mehrdad R. Health system in Iran. Japan Medical Association Journal. 2009; 52(1):69-73. https://www.researchgate.net/ profile/Ramin-Mehrdad/publication/232426386_Health_System_in_Iran/links/0c9605281d9d29d0bc000000/Health-Systemin-Iran.pdf\#page $=71$

[2] Naeli J, Mogimi D. Primary care health promotion policy document. Tehran: Ministry of Health; 2007.

[3] Bagyani-Mogadam H, Ehraampoosh M. Principles of health services. Tehran: Cherag-E Danesh; 2003.

[4] Park K. Park's textbook of preventive and social medicine. Jabalpur, Madhya Pradesh: Bhanot Publishers; 2017. https://books. google.com/books?id=SymHtAEACAAJ\&dq=Park\%E2\%80\%99 s+textbook + of + preventive + and + social + medicine $\&$ hl $=$ en\&sa $=X$ \&ved=2ahUKEwiyq7Ojp8nxAhWox4UKHYyOA3gQ6AEwAH oECAsQAQ

[5] Funk M. Integrating mental health into primary care: A global perspective [Internet]. 2008 [Updated 2008]. Available from: https://www.who.int/mental_health/policy/services/integratingmhintoprimarycare/en/

[6] Asadi-Lari M, Sayyari A, Akbari M, Gray D. Public health improvement in Iran-lessons from the last 20 years. Public Health. 2004; 118(6):395-402. [DOI:10.1016/j.puhe.2004.05.011] [PMID]

[7] Bagheri-Yazdi S, Malek-Afzali H, Shahmohammadi D, NaghaviRavandi M, Hekmat S. Evaluation of functions of auxiliary health workers (Behvarzes) and health volunteers in mental health care delivery in the framework of PHC system in Brojen city, Chaharmahal and Bakhtiary province. Hakim Research Journal. 2001; 4(2):100-9.

[8] Shahmohammadi D, Bayanzadeh S A, Ehssanmanesh M. [Pathways to psychiatric care in Iran (Persian)]. Iranian Journal of Psychiatry and Clinical Psychology (IJPCP). 1998; 3(4):4-14. http:/ / ijpcp.iums.ac.ir/article-1-1706-en.html

[9] CaracciG. Urban mental health: An international survey. International Journal of Mental Health. 2006; 35(1):39-45. [DOI:10.2753/ IMH0020-7411350103]

[10] Sharifi V, Abolhasani F, Farhoudian A, Amin-Esmaeili M. Community Mental Health Centers in Iran: Planning evidencebased services. Iranian Journal of Psychiatry \& Clinical Psychology. 2013; 19(3):163-76. https:// web.a.ebscohost.com/abstract?d irect $=$ true\&profile $=$ ehost\&scope $=$ site\&authtype $=$ crawler\&jrnl $=$

[11] Rashedi V, Malakouti K, Rudnik A. Community mental health service for older adults in Iran. Journal of Kermanshah University of Medical Sciences. 2018; 22(2):e78947. [DOI:10.5812/ jkums.78947]

[12] WHO and AIMS. A report of the assessment of the mental health system in Uttarkhand, India, using the World Health Organization - Assessment Instrument for Mental Health Systems (WHO-AIMS). Geneva: WHO 2006. https://apps.who.int/iris/ bitstream/handle/10665/206158/B0768.pdf?sequence=1

[13] Sharifi V. [Urban mental health in Iran: challenges and future directions (Persian)]. Iranian Journal of Psychiatry and Behavioral Sciences. 2009; 3(1):9-14. https://www.sid.ir/en/journal/ViewPaper.aspx?ID=143475

[14] Noorbala AA, Bagheri Yazdi SA, Asadi Lari M, Vaez Mahdavi MR. [Mental health status of individuals fifteen years and older in Tehran-Iran (2009) (Persian)]. Iranian Journal of Psychiatry and Clinical Psychology. 2011; 16(4):479-83. http://ijpcp.iums.ac.ir/ article-1-1212-en.html

[15] Noorbala AA, Yazdi SB, Yasamy M, Mohammad K. Mental health survey of the adult population in Iran. The British Journal of Psychiatry. 2004; 184(1):70-3. [DOI:10.1192/bjp.184.1.70] [PMID]

[16] Ministry of Health and Medical Education. A national report on registration of case with psychiatric disorder in integration of mental health in primary care program. Tehran: Ministry of Health and Medical Education; 2008. [Linke not Found]

[17] Amini H, Sharifi V, Nejatisafa A, Arbabi M, Tabatabaie M, Alimadadi $Z$, et al. [One year follow-up of patients with bipolar disorder admitted to Roozbeh Hospital (Persian)]. Iranian Journal of Psychiatry and Clinical Psychology. 2009; 15(2):168-74. http://ijpcp.iums.ac.ir $/$ browse.php?a_id=699\&slc_lang $=$ fa\&sid $=1 \& f t x t=1$

[18] Bolhari J, Zojaji A, Karimi-Kisomi I, Nazari-Jeirani M, Tabaee S. [Urban mental health service: Primary health care model with community participation. Iranian Journal of Psychiatry and Clinical Psychology. 2011; 17(2):110-5. http://irisweb.ir/files/site1/ rds_journals/21/article-21-85192.pdf

[19] Hajebi A, Damari B, Moghaddam AV, Nasehi A, Nikfarjam A Bolhari J. What to do to promote mental health of the society. Iranian Journal of Public Health. 2013; 42(Supple1):105-12. [PMCID] [PMID]

[20] Khayatzadeh A, Takian A. Family physician program in Iran: Considerations for adapting the policy in urban settings. Archives of Iranian Medicine. 2014; 17(11):776-778. [PMID]

[21] World Heald Organization. Improving health systems and services for mental health [Internet]. 2009 [Updated 2009]. Available from: http://apps.who.int/iris/bitstream/handle/10665/44219/9789241598774_eng.pdf;jsessionid=A91BCF97 C36BA33024CAF66A5A1881BE? sequence $=1$

[22] World Health Organization. Integrating mental health into primary care: A global perspective [Internet]. 2008 [Updated 2008]. Available from: https://www.who.int/mental_health/policy/ services/integratingmhintoprimarycare/en/

[23] World Health Organization. Investing in mental health. Geneva: World Health Organization. https://apps.who.int/iris/ handle/10665/42823

[24] State Welfare Organization of Iran. Social emergency program (Persian) [Internet]. 2020 [Updated 2020 September 27]. Available from: https://www.behzisti.ir/news/23534/

[25] Ministry of Cooperatives, Labour, and Social Welfare. Socia emergency program (Persian) [Internet]. 2021 [Updated 2021 July 04]. Available from: https://asibha.mcls.gov.ir/fa/ghorop/marriageanddirorce/manabeejtemaei/barname

[26] State Welfare Organization of Iran. Executive instructions of the Crisis Intervention Center (individual, family and social) "Social Emergency Center" (Persian) [Internet]. 2008 [Updated 2008]. Available from: http://hormozganbeh.ir/attachments/ article/2311/

[27] Gould MS, Kalafat J, HarrisMunfakh JL, Kleinman M. An evaluation of crisis hotline outcomes part 2: Suicidal callers. Suicide and Life-Threatening Behavior. 2007; 37(3):338-52. [DOI:10.1521/ suli.2007.37.3.338] [PMID] 
[28] Kalafat J, Gould MS, Munfakh JLH, Kleinman M. An evaluation of crisis hotline outcomes. Part 1: Nonsuicidal crisis callers. Suicide and Life-Threatening Behavior. 2007; 37(3):322-37. [DOI:10.1521/suli.2007.37.3.322] [PMID]

[29] Gilat I, Shahar G. Emotional first aid for a suicide crisis: comparison between Telephonic hotline and internet. Psychiatry: Interpersonal and Biological Processes. 2007; 70(1):12-8. [DOI:10.1521/ psyc.2007.70.1.12] [PMID]

[30] Bennett L, Riger S, Schewe P, Howard A, Wasco S. Effectiveness of hotline, advocacy, counseling, and shelter services for victims of domestic violence: A statewide evaluation. Journal of Interpersonal Violence. 2004; 19(7):815-29. [DOI:10.1177/0886260504265687] [PMID]

[31] Johnson S, Nolan F, Pilling S, Sandor A, Hoult J, McKenzie N, et al. Randomised controlled trial of acute mental health care by a crisis resolution team: the north Islington crisis study. The BMJ. 2005; 331(7517):599. [DOI:10.1136/bmj.38519.678148.8F] [PMID] [PMCID]

[32] Irving CB, Adams CE, Rice K. Crisis intervention for people with severe mental illnesses. Cochrane Database of Systematic Reviews. 2006; 4. [DOI:10.1002/14651858.CD001087.pub3]

[33] Jacobs R, Barrenho E. Impact of crisis resolution and home treatment teams on psychiatric admissions in England. The British Journal of Psychiatry. 2011; 199(1):71-6. [DOI:10.1192/bjp. bp.110.079830] [PMID]

[34] Needle J, Bindman JP, Thornicroft G. Crisis resolution and home treatment in mental health. Johnson S, editor. Cambridge: Cambridge University Press; 2008. [DOI:10.1017/S003329170999170X]

[35] McGlynn P, editor. Crisis resolution and home treatment: A practical guide. London: Sainsbury Centre for Mental Health; 2006.

[36] Farhoudian A, Sharifi V, Amini H, Basirnia A, Mesgarpour B, Mansouri N, et al. Prevalence of psychiatric disorders in Iran: A systematic review. Iranian Journal of Psychiatry. 2007; 2(4):137-50. https://www.sid.ir/en/Journal/ViewPaper.aspx?ID=165135

[37] Malakouti SK, Nojomi M, Panaghi L, Chimeh N, Mottaghipour Y, Joghatai MT, et al. Case-management for patients with schizophrenia in Iran: a comparative study of the clinical outcomes of mental health workers and consumers' family members as case managers. Community Mental Health Journal. 2009; 45(6):447-52 [DOI:10.1007/s10597-009-9197-4] [PMID]

[38] Mansouri N, Malakouti SK, Chimeh N, Rezvanifar S, Mohseni M, Mansouri E. Chronic hospitalized and household maintained consumers; characteristics and differences among referees to the Iranian society supporting individuals with Schizophrenia. Iranian Journal of Psychiatry and Behavioral Sciences. 2014; 8(1):5-12. [PMCID] [PMID]

[39] Mansouri N, Chimeh N, Dehghani M, MalakoutiSK, Taherkhani H, Abarashi Z. Risk factors associated with psychiatric hospitalization among Iranian schizophrenic patients. Iranian Journal of Psychiatry and Behavioral Sciences. 2013; 7(2):16-23. [PMCID] [PMID]

[40] Malakouti SK, Mirabzadeh A, Nojomi M, Tonkaboni AA, Nadarkhani F, Mirzaie M, et al. Clinical outcomes and cost effectiveness of two aftercare models provided by general physicians and nurses to patients with severe mental illness. Medical Journal of the Islamic Republic of Iran. 2015; 29:196. [PMCID] [PMID]
[41] Khaleghparast S, Ghanbari B, Kahani S, Malakouti K, SeyedAlinaghi S, Sudhinaraset M. The effectiveness of discharge planning on the knowledge, clinical symptoms and hospitalisation frequency of persons with schizophrenia: a longitudinal study in two hospitals in Tehran, Iran. Journal of Clinical Nursing. 2014; 23(15-16):2215-22. [DOI:10.1111/jocn.12499] [PMID]

[42] Moradi-Lakeh M, Yaghoubi M, Hajebi A, Malakouti SK, Vasfi MG. Cost-effectiveness of aftercare services for people with severe mental disorders: An analysis parallel to a randomised controlled clinical trial in Iran. Health \& Social Care in the Community. 2017; 25(3):1151-9 [DOI:10.1111/hsc.12416] [PMID]

[43] Sharifi V, Amini H, Tehrani DM, Yasami M, Jalali RM, Sobh BP, et al. Roozbeh home care program for severe mental disorders: A preliminary report. Iranian Journal of Psychiatry. 2006; 1(1):31-4. https://ijps.tums.ac.ir/index.php/ijps/article/view/400/395

[44] Glick ID, Fleming L, DeChillo N, Meyerkopf N, Jackson C, Muscara D, et al. A controlled study of transitional day care for non-chronically-ill patients. The American Journal of Psychiatry. 1986; 143(12):1551-6. [DOI:10.1176/ajp.143.12.1551] [PMID]

[45] Linn MW, Caffey EM, Klett CJ, Hogarty GE, Lamb HR. Day treatment and psychotropic drugs in the aftercare of schizophrenic patients: A veterans administration cooperative study. Occupational Therapy in Mental Health. 1980; 1(1):77-106. [DOI:10.1300/ J004v01n01_06

[46] Wiersma D, Kluiter H, Nienhuis FJ, Rüphan M, Giel R. Costs and benefits of day treatment with community care for schizophrenic patients. Schizophrenia Bulletin. 1991; 17(3):411-9. [DOI:10.1093/schbul/17.3.411] [PMID]

[47] Creed F, Black D, Anthony P, Osborn M, Thomas P, Tomenson B. Randomised controlled trial of day patient versus inpatient psychiatric treatment. The BMJ. 1990; 300(6731):1033-7. [DOI:10.1136/bmj.300.6731.1033] [PMID] [PMCID]

[48] Štrkalj-Ivezić S, Vrdoljak M, Mužinić L, Agius M. The impact of a rehabilitation day centre program for persons suffering from schizophrenia on quality of life, social functioning and self-esteem. Psychiatria Danubina. 2013; 25(2):194-9. http://www.psychiatria-danubina.com/UserDocsImages/pdf/dnb_vol25\%20 Suppl\%202_no/dnb_vol25_noSuppl\%202_194.pdf

[49] Marshall M, Crowther R, Almaraz-Serrano A, Creed F, Sledge $\mathrm{W}$, Kluiter $\mathrm{H}$, et al. Systematic reviews of the effectiveness of day care for people with severe mental disorders: (1) acute day hospital versus admission; (2) vocational rehabilitation; (3) day hospital versus outpatient care. Health technology assessment (Winchester, England). 2001; 5(21):1-75. [DOI:10.3310/hta5210] [PMID]

[50] Lloyd C. Vocational rehabilitation and mental health. Hoboken: John Wiley \& Sons; 2010. [DOI:10.1002/9781444319736]

[51] Williams A, Fossey E, Harvey C. Sustaining employment in a social firm: use of the Work Environment Impact Scale v 2.0 to explore views of employees with psychiatric disabilities. British Journal of Occupational Therapy. 2010; 73(11):531-9. [DOI:10.4276 /030802210X12892992239279]

[52] Warner R. The diffusion of two successful rehabilitation models. World Psychiatry. 2006; 5(3):160-1. [PMCID] [PMID]

[53] Kubiak S, Roddy J, Comartin E, Tillander E. Cost analysis of long-term outcomes of an urban mental health court. Evaluation and Program Planning. 2015; 52:96-106 [DOI:10.1016/j.evalprogplan.2015.04.002] [PMID] 
[54] Wells R, Kite B, Breckenridge E, Sunbury T. Community mental health center integrated care outcomes. Psychiatric Quarterly. 2018; 89(4):969-82. [DOI:10.1007/s11126-018-9594-3] [PMID]

[55] McKay C, Johnsen M, Stein R. Employment outcomes in Massachusetts clubhouses. Psychiatric Rehabilitation Journal. 2005; 29(1):25-33. [DOI:10.2975/29.2005.25.33] [PMID]

[56] Auerbach ES. The individual placement and support model vs. the menu approach to supported employment: Where does Occupational Therapy fit in? Occupational Therapy in Mental Health. 2002; 17(2):1-19. [DOI:10.1300/J004v17n02_01]

[57] Moll S, Huff J, Detwiler L. Supported employment: Evidence for a best practice model in psychosocial rehabilitation. Canadian Journal of Occupational Therapy. 2003; 70(5):298-310. [DOI:10.117 7/000841740307000506] [PMID]

[58] Crowther RE, Marshall M, Bond GR, Huxley P. Helping people with severe mental illness to obtain work: Systematic review. The BMJ. 2001; 322(7280):204-8. [DOI:10.1136/bmj.322.7280.204] [PMID] [PMCID]

[59] Lehman AF, Goldberg R, Dixon LB, McNary S, Postrado L, Hackman A, et al. Improving employment outcomes for persons with severe mental illnesses. Archives of General Psychiatry. 2002; 59(2):165-72. [DOI:10.1001/archpsyc.59.2.165] [PMID]

[60] Corrigan PW, Mueser KT, Bond GR, Drake RE, Solomon P. Principles and practice of psychiatric rehabilitation: An empirical approach. New York: Guilford Press; 2012.

[61] Rogers ES, Anthony W, Kash M, Olschewski A. Systematic review of supported housing literature 1993-2008. Center for Psychiatric Rehabilitation. Boston: Boston University; 2010.

[62] Tsemberis S. Housing first: The pathways model to end homelessness for people with mental illness and addiction manual. European Journal of Homelessness. 2011; 5(2):235-40. https:/ / www. researchgate.net/publication/47669330_

[63] Chilvers R, Macdonald G, Hayes A. Supported housing for people with severe mental disorders. Cochrane Database of Systematic Reviews. 2006; (4):CD000453 [DOI:10.1002/14651858. CD000453.pub2] [PMID] [PMCID]

[64] Malakouti SK, Noori R, Naghavi M, Ahmad Abhari A, Nasr M, \& Afagh S, Salehi P, et al. [The burden of caregivers of chronic mental patients and their needs to care and therapeutic services (Persian)]. Hakim. 2003; 6(2):1-10. https://www.sid.ir/en/Journal/ViewPaper.aspx?ID=62559

[65] Damari B, Alikhani S, Riazi-Isfahani S, Hajebi A. Transition of mental health to a more responsible service in Iran. Iranian Journal of Psychiatry. 2017; 12(1):36-41. [PMCID] [PMID] 
This Page Intentionally Left Blank 